HUTP-01/A023

HU-EP-01/21

hep-th/0105045

\title{
Disk Instantons, Mirror Symmetry and the Duality Web
}

\author{
Mina Aganagic ${ }^{1}$, Albrecht Klemm² and Cumrun Vafa $^{1}$ \\ 1 Jefferson Physical Laboratory \\ Harvard University, Cambridge, MA 02138, USA \\ ${ }^{2}$ Institut für Physik, Humboldt Universität zu Berlin \\ Invaliden Straße 110, D-10115, Germany
}

\begin{abstract}
We apply the methods recently developed for computation of type IIA disk instantons using mirror symmetry to a large class of D-branes wrapped over Lagrangian cycles of non-compact Calabi-Yau 3-folds. Along the way we clarify the notion of "flat coordinates" for the boundary theory. We also discover an integer IR ambiguity needed to define the quantum theory of D-branes wrapped over non-compact Lagrangian submanifolds. In the large $N$ dual Chern-Simons theory, this ambiguity is mapped to the UV choice of the framing of the knot. In a type IIB dual description involving $(p, q) 5$-branes, disk instantons of type IIA get mapped to $(p, q)$ string instantons. The M-theory lift of these results lead to computation of superpotential terms generated by M2 brane instantons wrapped over 3-cycles of certain manifolds of $G_{2}$ holonomy.
\end{abstract}

May 2001 


\section{Introduction}

D-branes wrapped over non-trivial cycles of a Calabi-Yau threefold provide an interesting class of theories with 4 supercharges (such as $N=1$ supersymmetric theories in $d=4)$. As such, they do allow the generation of a superpotential on their worldvolume. This superpotential depends holomorphically on the chiral fields which parameterize normal deformations of the wrapped D-brane.

On the other hand F-terms are captured by topological string amplitudes [1] and in particular the superpotential is computed by topological strings at the level of the disk amplitude [1] [2] [3] [4]. More generally the topological string amplitude at genus $g$ with $h$ holes computes superpotential corrections involving the gaugino superfield $W$ and the $N=2$ graviphoton multiplet $\mathcal{W}$ given by $h \int d^{2} \theta\left(\operatorname{Tr} \mathrm{W}^{2}\right)^{\mathrm{h}-1}\left(\mathcal{W}^{2}\right)^{\mathrm{g}}$ [5]. So the issue of computation of topological string amplitudes becomes very relevant for this class of supersymmetric theories.

In the context of type IIA superstrings such disk amplitudes are given by non-trivial worldsheet instantons, which are holomorphic maps from the disk to the CY with the boundary ending on the D-brane. Such computations are in general rather difficult. The same questions in the context of type IIB strings involve classical considerations of the worldsheet theory. In a recent paper [6] it was shown how one can use mirror symmetry in an effective way to transform the type IIA computation of disk instantons to classical computations in the context of a mirror brane on a mirror CY for type IIB strings. The main goal of this paper is to extend this method to more non-trivial Calabi-Yau geometries.

One important obstacle to overcome in generalizing [6] is a better understanding of "flat coordinates" associated with the boundary theory, which we resolve by identifying it with BPS tension of associated domain walls. We also uncover a generic IR ambiguity given by an integer in defining a quantum Lagrangian D-brane. We relate this ambiguity to the choice of the regularizations of the worldsheet theory associated to the boundaries of moduli space of Riemann surfaces with holes (the simplest one being two disks connected by an infinite strip). In the context of the Large $N$ Chern-Simons dual [7] applied to Wilson Loop observables [4] this ambiguity turns out to be related to the UV choice of the framing of the knot, which is needed for defining the Wilson loop observable by point splitting [8].

Along the way, for gaining further insight, we consider other equivalent dual theories, including the lift to M-theory, involving M-theory in a $G_{2}$ holonomy background. In this 
context we are able to transform the generation of superpotential by Euclidean M2 branes (with the topology of $S^{3}$ ) to disk instantons of type IIA 1 for M-theory on $G_{2}$ holonomy manifolds and use mirror symmetry to compute them! We also relate this theory to another dual type IIB theory in a web of $(p, q)$ 5-branes in the presence of ALF-like geometries.

The organization of this paper is as follows: In section 2 we review the basic setup of [6]. In section 3 we consider the lift of these theories to M-theory in the context of $G_{2}$ holonomy manifolds, as well as to type IIB theory with a web of $(p, q)$ 5-branes in an ALF-like background. In section 4 we identify the flat coordinates for boundary fields by computing the BPS tension of D4 brane domain walls ending on D6 branes wrapping Lagrangian submanifolds. In section 5 we discuss the integral ambiguity in the computation of topological string amplitudes and its physical meaning. This is discussed both in the context of Large N Chern-Simons/topological string duality, as well as in the context of the type IIB theory with a web of $(p, q) 5$-branes. In section 6 we present a large class of examples, involving non-compact CY 3-folds where the D6 brane wraps a non-compact Lagrangian submanifold. In appendix A we perform some of the computations relevant for the framing dependence for the unknot and verify that in the large $N$ dual description this UV choice maps to the integral IR ambiguity we have discovered for the quantum Lagrangian D-brane.

\section{Review of Mirror Symmetry for D-branes}

In this section we briefly recall the mirror symmetry construction for non-compact toric Calabi-Yau manifolds (specializing to the case of threefolds), including the mirror of some particular class of (special) Lagrangian D-branes on them.

Toric Calabi-Yau threefolds arise as symplectic quotient spaces $X=\mathbf{C}^{3+k} / / G$, for $G=U(1)^{k}$. The quotient is obtained by imposing the $k D$-term constraints

$$
D^{a}=Q_{1}^{a}\left|X^{1}\right|^{2}+Q_{2}^{a}\left|X^{2}\right|^{2}+\ldots Q_{3+k}^{a}\left|X^{3+k}\right|^{2}-r^{a}=0
$$

where $a=1, \ldots k$, and dividing by $G$

$$
X^{i} \rightarrow e^{i Q_{i}^{a} \epsilon_{a}} X^{i}
$$

1 More generally we can map the generation of superpotential-like terms associated to topological strings at genus $g$ with $h$ boundaries to Euclidean M2 brane instantons on a closed 3-manifold with $b_{1}=2 g+h-1$. 
The $c_{1}(X)=0$ condition is equivalent to $\sum_{i} Q_{i}^{a}=0$. The Kähler structure is encoded in terms of the $r^{a}$ and varying them changes the sizes of various 2 and 4 cycles. In the linear sigma model realization [9] this is realized as a $(2,2)$ supersymmetric $U(1)^{k}$ gauge theory with $3+k$ matter fields $X^{i}$ with charges given by $Q_{i}^{a}$, and with $k$ FI terms for the $U(1)^{k}$ gauge group given by $r^{a}$.

The mirror theory is given in terms of $n+k$ dual $\mathbf{C}^{*}$ fields $Y^{i}$ [10], where

$$
\operatorname{Re}\left(Y^{i}\right)=-\left|X^{i}\right|^{2}
$$

with the periodicity $Y^{i} \sim Y^{i}+2 \pi i$. The D-term equation (2.1) is mirrored by

$$
Q_{1}^{a} Y^{1}+Q_{2}^{a} Y^{2}+\ldots Q_{3+k}^{a} Y^{3+k}=-t^{a}
$$

where $t^{a}=r^{a}+i \theta^{a}$ and $\theta^{a}$ denotes the $\theta$-angles of the $U(1)^{a}$ gauge group. Note that (2.4) has a three-dimensional family of solutions. One parameter is trivial and is given by $Y^{i} \rightarrow Y^{i}+c$. Let us pick a parameterization of the two non-trivial solutions by $u, v$.

The mirror theory can be represented as a theory of variations of complex structures of a hypersurface $Y$

$$
x z=e^{Y^{1}(u, v)}+\ldots+e^{Y^{k+3}(u, v)} \equiv P(u, v)
$$

where

$$
Y^{i}(u, v)=a^{i} u+b^{i} v+t^{i}(t)
$$

is a solution to (2.4) (in obtaining this form, roughly speaking the trivial solution of shifting of all the $Y^{i}$ has been replaced by $x, z$ whose product is given by the above equation). We choose the solutions so that the periodicity condition of the $Y^{i} \sim Y^{i}+2 \pi i$ are consistent with those of $u, v$ and that it forms a fundamental domain for the solution. Note that this in particular requires $a^{i}, b^{i}$ to be integers. Even after taking these constrains into account there still is an $\mathrm{SL}(2, \mathbb{Z})$ group action on the space of solutions via

$$
\begin{aligned}
& u \rightarrow a u+b v \\
& v \rightarrow c u+d v .
\end{aligned}
$$

Note that the holomorphic 3-form for CY is given by

$$
\Omega=\frac{\mathrm{d} x \mathrm{~d} u \mathrm{~d} v}{x},
$$

and is invariant under the $\operatorname{SL}(2, \mathbb{Z})$ action. 


\subsection{Special Lagrangian Submanifolds and Mirror Branes}

In [6] a family of special Lagrangian submanifolds of the A-model geometry was studied, characterized by two charges $q_{i}^{\alpha}$ with $i=1, \ldots, k+3$ and $\alpha=1,2$, subject to

$$
\sum_{i} q_{i}^{\alpha}=0
$$

and in terms of which the Lagrangian submanifold is given by three constrains. Two of them given by

$$
\sum q_{i}^{\alpha}\left|X^{i}\right|^{2}=c^{\alpha}
$$

and the third is $\sum \theta^{i}=0$ where $\theta^{i}$ denotes the phase of $X^{i}$. The worldsheet boundary theory for this class of theories has been further studied in [11].

The submanifolds in question project to one dimensional subspaces of the toric base (taking into account the constrains $(2.1),(2.7)$ ),

$$
\left|X^{i}\right|^{2}=r+b^{i}
$$

for some fixed $b^{i}$ (depending on $c^{\alpha}, r^{a}$ ) and $r \in \mathbf{R}^{+}$. In order to get a smooth Lagrangian submanifold one has to double this space (by including the $\sum \theta^{i}=\pi$ ). The topology of the Lagrangian submanifold is $R \times S^{1} \times S^{1}$. There is however a special choice of $c^{\alpha}$ which makes the Lagrangian submanifold pass through the intersection line of two faces of the toric base. The topology of the Lagrangian submanifold will be different in this limit. It corresponds to having one of the $S^{1}$ cycles pinched at a point of $R$ in the Lagrangian submanifold. This is topologically the same as two copies of $\mathbf{C} \times S^{1}$ touching at the origin of $\mathbf{C}$. In this limit we view the Lagrangian submanifold as being made of two distinct ones intersecting over an $S^{1}$. We can now have a deformation, which moves the two Lagrangian submanifolds independently, where the end point of each one should be a point (not necessarily the same) on the base of the toric geometry (see the example below).

Under mirror symmetry, the A-brane maps to a holomorphic submanifold of the $Y$ given by

$$
x=0=P(u, v)=e^{Y^{1}(u, v)}+\ldots+e^{Y^{3+k}(u, v) .}
$$

The mirror brane is one-complex dimensional, and is parameterized by $z$. Its moduli space is one complex dimensional parameterized by a point on a Riemann surface $P(u, v)=0$. The choice of the point depends on $c^{\alpha}$ and the Wilson line around $S^{1}$ and it is possible to read it off in the weak coupling limit of large volume of Calabi-Yau and large parameters $c^{\alpha}$ as discussed in [6]. 


\subsection{Example}

For illustration consider $X=O(-1) \oplus O(-1) \rightarrow P^{1}$, which is also called small resolution of conifold. This sigma model is realized by $U(1)$ gauge theory with 4 chiral fields, with charges $Q=(1,1,-1,-1)$. The D-term potential vanishes on $\left|X^{1}\right|^{2}+\left|X^{2}\right|^{2}-\left|X^{3}\right|^{2}-$ $\left|X^{4}\right|^{2}=r$, and $X$ is a quotient of this by $U(1)$. The D-term equations can be regarded as linear equations by projecting $X^{i} \rightarrow\left|X^{i}\right|^{2}$, and solved graphically in the positive octant of $R^{3}$ (see Fig. 1).

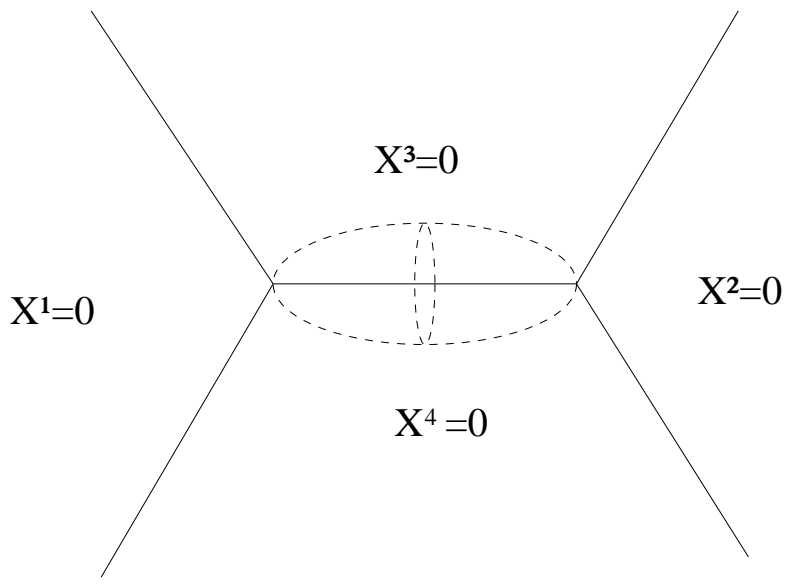

Fig.1 $X=O(-1) \oplus O(-1) \rightarrow \mathbf{P}^{\mathbf{1}}$ viewed as a toric fibration. The base is $\left(\left|X^{1}\right|^{2},\left|X^{3}\right|^{2},\left|X^{4}\right|^{2}\right)$ as generic solution to the vanishing of D-term potential, but is bounded by $\left|X^{2}\right|^{2} \geq 0$ hyperplane. Over the faces of the bounding hyperplanes some cycles of the fiber shrink. For example, there is a minimal $\mathbf{P}^{\mathbf{1}}$ in $X$ which lies over the finite edge.

$X$ is fibered over this base with fiber which is torus of phases of $X^{i}$ 's modulo $U(1), T^{3}=T^{4} / U(1)$. Note that $r$ is the size of a minimal $\mathbf{P}^{\mathbf{1}}$ at $X^{3}=0=X^{4}$.

Consider a special Lagrangian D-brane in this background with $q_{1}=(1,0,0,-1)$, $q_{2}=(0,0,1,-1)$. This gives two constrains $\left|X^{1}\right|^{2}-\left|X^{4}\right|^{2}=c_{1}$ and $\left|X^{3}\right|^{2}-\left|X^{4}\right|^{2}=c_{2}$ in the base which determine a two dimensional family of Lagrangians, but D-branes of topology $\mathbf{C} \times \mathbf{S}^{\mathbf{1}}$ are further constrained to live on the one dimensional faces of the base. For this we need for example $c_{2}=0$, and $c_{1}$ arbitrary but in $(0, r)$ interval. As discussed above this can be viewed as coming from the deformation of a Lagrangian 
submanifold which splits to two when it intersects the edges of the toric geometry and move them independently on the edge. See Fig. 2. Typically we would be interested in varying the position of one brane, keeping the other brane fixed (or taken to infinity along an edge).

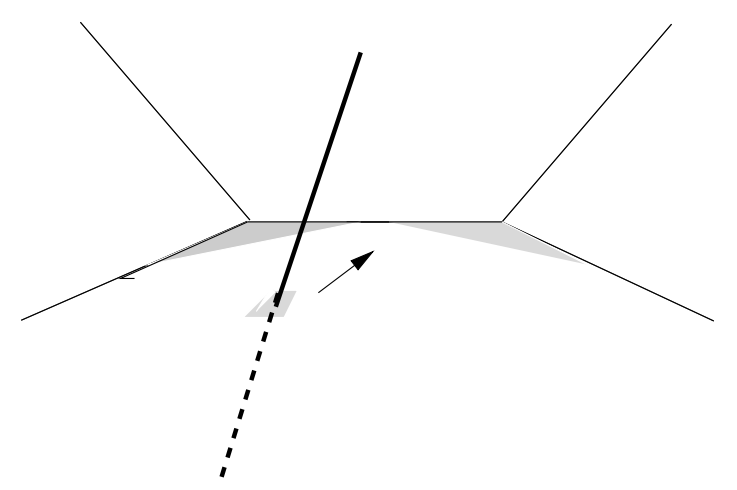

a.

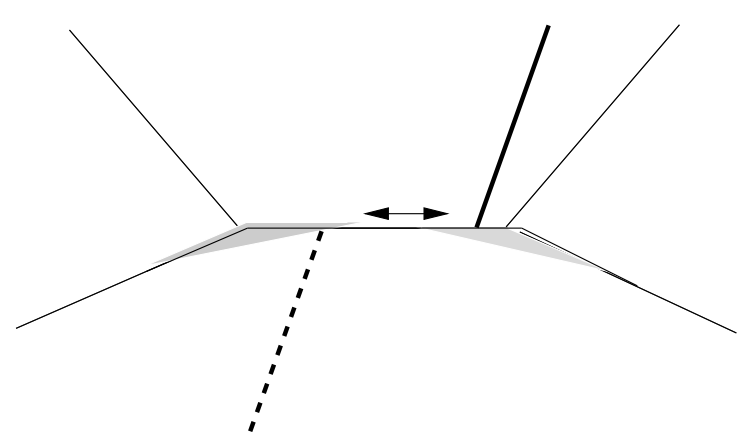

c.

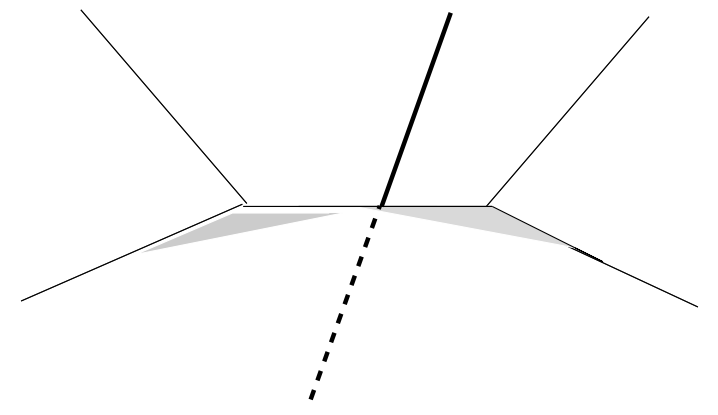

b.

Fig.2 The special Lagrangian submanifold which has topology $R \times T^{2}$ for generic values of $c_{i}$ (case a) can degenerate (case b) and split (case c) into two Lagrangian submanifolds, when it approaches a one-dimensional edge of the toric base. The two resulting components have topology $\mathbf{C} \times \mathbf{S}^{\mathbf{1}}$, and can move independently, but only along one-dimensional edges.

The mirror of $X$ is

$$
x z=e^{u}+e^{v}+e^{-t-u+v}+1
$$

obtained by solving $Y^{1}+Y^{2}-Y^{3}-Y^{4}=-t$ for $Y^{2}$, fixing the trivial solution by setting $Y^{4}=0$, and putting $Y^{1}=u$ and $Y^{3}=v$.

The mirror B-brane propagates on the Riemann surface $0=P(u, v)=e^{u}+e^{v}+$ $e^{-t-u+v}+1$ shown in Figure 3 . 


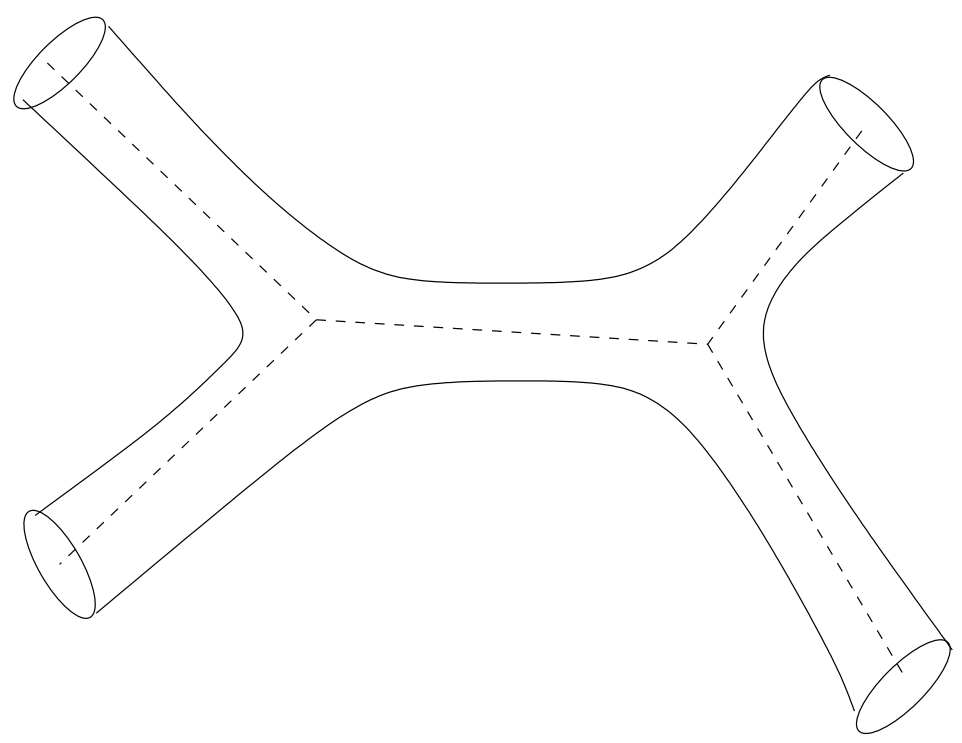

Fig.3 Riemann surface $\Sigma: P(u, v)=0$ corresponding to the mirror of $X=O(-1) \oplus$ $O(-1) \rightarrow \mathbf{P}^{\mathbf{1}} . \Sigma$ is related to the toric diagram of $X$ by thickening out the one-dimensional edges of the base in Fig.1.

Note that mirror map (2.3) gives the B-brane at $\operatorname{Re}(u)=-c_{1}$ and $\operatorname{Re}(v)=0$ which is on the Riemann surface in the large radius limit, $r \gg 0$ and $r / 2>c_{1} \gg 0$. In other words, in the large radius limit, classical geometry of the D-brane moduli space is a good approximation to the quantum geometry given by $\Sigma$.

We can also construct, as a limit, Lagrangian submanifolds of $\mathbf{C}^{3}$ by considering the limit $r+i \theta=t \rightarrow \infty$ holding $c_{1}$ fixed, as shown in figure 4 . In this limit the mirror geometry become $x z=e^{u}+e^{v}+1$.

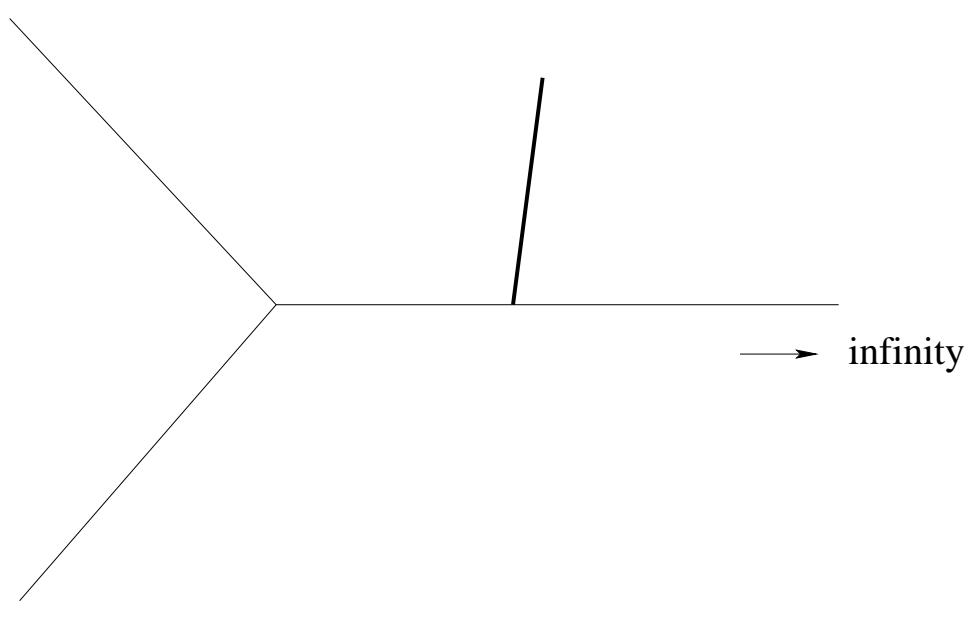


Fig.4 In the limit in which the size $t$ of the $\mathbf{P}^{\mathbf{1}}$ in $X=O(-1) \oplus O(-1) \rightarrow \mathbf{P}^{\mathbf{1}}$ goes to infinity, the manifold looks locally like $\mathbf{C}^{\mathbf{3}}$, together with a Lagrangian D-brane.

This case was studied in detail in [12].

\subsection{Disk Amplitude}

The disk amplitudes of the topological A-model give rise to an $N=1$ superpotential in the corresponding type IIA superstring theory [1] [2] [3] [4] where we view the D6 brane as wrapping the Lagrangian submanifold and filling the spacetime. The corresponding superpotential for the mirror of the Lagrangian submanifolds we have discussed above was computed in [6], and is given in terms of the Abel-Jacobi map

$$
W(u)=\int_{u_{*}}^{u} v(u) d u .
$$

where $u_{*}$ is some fixed point on the Riemann surface $P(u, v)=0$ and the line integral is done on this surface. This defines the superpotential up to an addition of a constant. More physically if we construct the 'splitting' of the Lagrangian brane over the toric edges, we can view $u_{*}$ as the location of one of the Lagrangian halves, which we consider fixed.

Note that if we move the point $u$ on the Riemann surface over a closed cycle and come back to the same point, the superpotential (2.10) may change by an overall shift, which depends on the choice of the cycle as well as the moduli of the Riemann surface (given by $t$ 's). It is natural to ask what is the interpretation of this shift. This shift in superpotential can be explained both from the viewpoint of type IIA and type IIB. In the context of type IIA this corresponds to taking the Lagrangian D6 brane over a path, whose internal volume traces a 4-dimensional cycle $C_{4}$ of $\mathrm{CY}$ (fixing the boundary conditions at infinity). By doing so we have come back to the same Brane configuration, but in the process we have shifted the RR 2-form flux. The 4-cycle $C_{4}$ is dual to a 2 -form which we identify with the shift in the RR 2form flux. In the type IIA setup this process changes the superpotential by (the

quantum corrected) $\int_{C_{4}} k \wedge k$, as discussed in [13] [14]. The Type IIB version of this involves varying D5 brane wrapped over a 2-cycle over a path and bringing it back to the original place. During this process the brane traces a 3-cycle in the internal Calabi-Yau which contributes integral of the holomorphic 3-form $\Omega$ over the 
3-cycle to the superpotential. This is interpreted as shifting the RR flux of $H$ along the dual 3-cycle. Note that we can use this idea to generate fluxes by bringing in branes not intersecting the toric edge, to the edges, splitting them on the edge and bringing it back together and then moving it off the toric edge. The process leads to the same CY but with some RR flux shifted.

The superpotential (2.10) is not invariant under different choices of parameterization of the fundamental domain for $u, v$ given by an $\mathrm{SL}(2, \mathbb{Z})$ transformation, but transforms as

$$
\begin{gathered}
W(u) \rightarrow W(u)+\int d\left[a c u^{2} / 2+b d v^{2} / 2-b c u v\right]= \\
W(u)+a c u^{2} / 2+b d v^{2} / 2-b c u v
\end{gathered}
$$

where $v$ is defined implicitly in terms of $u$ by $P(u, v)=0$. Note that if we added a boundary term it could have canceled this change in superpotential, which can be viewed as a choice of boundary condition at infinity on the non-compact brane [6]. Thus this IR choice is needed for the definition of the brane, and as we see it affects the physics by modifying the superpotential. As discussed in [6] the choice of the splitting to $u, v$ depends on the boundary conditions at infinity on the fields normal to the brane. Each $\mathrm{SL}(2, \mathbb{Z})$ action picks a particular choice of boundary conditions on the D-brane. Using the mirror symmetry and what A-model is computing, below we will be able to fix a canonical choice, up to an integer, which we will interpret physically.

As noted above, in terms of the topological A-model, superpotential $W$ is generated by the disk amplitudes. The general structure of these amplitudes has been determined in [4] where it was found that

$$
W=\sum_{k, n, \vec{m}} \frac{1}{n^{2}} N_{k, \vec{m}} \exp (n[k u-\vec{m} \cdot \vec{t}])
$$

Here $u$ parameterizes the size of a non-trivial holomorphic disk and where $N_{k, \vec{m}}$ are integers capturing the number of domain wall $D 4$ branes ending on the $D 6$ brane, which wrap the CY geometry in the 2-cycle class captured by $\vec{m}$, and $k$ denotes the wrapping number around the boundary.

In the large volume limit (where the area of 2-cycles ending or not ending on the D-brane are large) the A-model picture is accurate enough. In this case we do not expect a classical superpotential as there is a family of special Lagrangian 
submanifolds. Since $d W / d u=v$ and $W$ should be zero for any moduli of the brane, we learn that $v=0$ on the brane. This in particular chooses a natural choice of parameterization of the curve adapted to where the brane is. In particular the Dbrane is attached to the line which is classically specified by $v=0$ (which can always be done). $u$ should be chosen to correspond to the area of a basic disk instanton. However this can be done in many ways. In particular suppose we have one choice of such $u$. Then

$$
u \rightarrow u+n v
$$

is an equally good choice, because $v$ vanishes on the Lagrangian submanifold in the classical limit. So even though this ambiguity by an integer is irrelevant in the classical limit, in the quantum theory since $v$ is non-vanishing due to worldsheet instanton corrections this dramatically changes the quantum answer. Thus we have been able to fix the $u, v$ coordinates up to an integer choice $n$ for each particular geometry of brane. We will discuss further the meaning of the choice of $n$ in section 3 and 4.

Later we will see that there is a further correction to what $u, v$ are quantum mechanically. In particular as we will discuss in section 3 this arises because the quantum area of the disk differs from the classical computation which gives $u$. This is similar to what happens for the closed string theory where the parameter $t$ which measures the area of the basic sphere is replaced by the quantum corrected area $T$. This is usually referred to as the choice of the "flat coordinates" for the Calabi-Yau moduli.

\section{3. $G_{2}$ holonomy and type IIB 5-brane Duals}

Consider type IIA superstrings on a non-compact Calabi-Yau threefold $X$ with a special Lagrangian submanifold $L \subset X$. Consider wrapping a D6 brane around $L$ and filling $R^{4}$. This theory has $N=1$ supersymmetry on $R^{4}$ and we have discussed the superpotential generated for this theory. In this section we would like to relate this to other dual geometries. 


\subsection{M-theory Perspective}

D6 branes are interpreted as KK monopoles of M-theory. This means that in the context of M-theory the theories under consideration should become purely geometric. This in fact was studied in [16][17] [18] [19] [20] where it was seen that the M-theory geometry corresponds to a 7 dimensional manifold with $G_{2}$ holonomy. In other words we consider a 7 -fold which is roughly $Y \sim X \times S^{1}$ where $S^{1}$ is fibered over the CY manifold $X$ and vanishes over the location of the Lagrangian submanifold $L \subset X$. In this context the superpotentials that we have computed must be generated by M2 brane instantons wrapping around non-trivial 3-cycles. Some examples of Euclidean M2 brane instantons for $G_{2}$ holonomy manifolds has been studied in [21. In fact there is a direct map from the disks ending on $L$ to a closed 3-cycle with the topology of $S^{3}$. In order to explain this we first discuss some topological facts about $S^{3}$.

We can view $S^{3}$ as

$$
\left|z_{1}\right|^{2}+\left|z_{2}\right|^{2}=1
$$

with $z_{i}$ complex numbers. Let $x=\left|z_{1}\right|^{2}$. The range for $x$ varies from 0 to 1 . There is an $S^{1} \times S^{1}$ of $S^{3}$ which project to any fixed $x$ with $0<x<1$, given by the phases of $z_{1}$ and $z_{2}$. At $x=0$ the circle corresponding to the phase of $z_{1}$ shrinks and at $x=1$ the circle corresponding to the phase of $z_{2}$ shrinks. So we can view the $S^{3}$ as the product of an interval with two $S^{1}$ 's where one $S^{1}$ shrinks at one end and the other $S^{1}$ shrinks at the other end. See Fig.5.

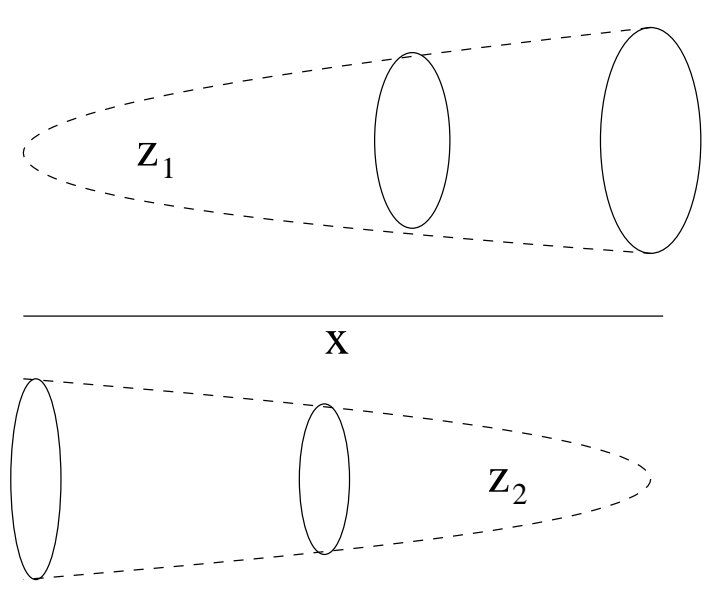


Fig.5 We can view $S^{3}$ as an $S^{1} \times S^{1}$ fibration over an interval. Near the ends of the interval it can be viewed as a complex plane $\mathbf{C} \times S^{1}$, where the complex plane is $z_{1}$ at one end and $z_{2}$ at the other. This gives two inequivalent descriptions of $S^{3}$ in terms of a circle fibered over a disk.

We can also view $S^{3}$ as a disk times a circle where the circle vanishes on one boundary-this can be done in two different ways, as shown in Fig.5.

Now we are ready to return to our case. Consider a disk of type IIA. The M2 brane Euclidean instanton can be viewed as the disk times an $S^{1}$, where the $S^{1}$ is the '11-th' circle. Note that on the boundary of the disk, which corresponds to the Lagrangian submanifold, the 11-th circle shrinks. Therefore, from our discussion above, this three dimensional space has the topology of $S^{3}$.

We have thus seen that using mirror symmetry, by mapping the type IIA geometry with a brane to an equivalent type IIB with a brane, and computing the superpotential, in effect we have succeeded in transforming the question of computation of superpotentials generated by M2 brane instantons in the context of $G_{2}$ holonomy manifolds, to an application of mirror symmetry in the context of D-branes! More generally, one can in principle compute, using mirror symmetry [22], partition function for higher genus Riemann surfaces with boundaries. This computes F-term cor-

rections to the spacetime theory [5] given by $h \int d^{4} x d^{2} \theta F_{g, h}\left(\mathcal{W}^{2}\right)^{g} \operatorname{Tr}\left(\mathrm{W}^{2}\right)^{h-1}$ where $\mathcal{W}$ is the gravi-photon multiplet, and $W$ the $N=1$ gaugino superfield containing the $U(M)$ field strength on the worldvolume of $\mathrm{M}$ coincident $K K$ monopoles (if we wish to get infinitely many such contributions we need $M \rightarrow \infty)$. It is easy to see that the topology of the corresponding M2 brane instantons is a closed 3-manifold with $b_{1}=2 g+h-1$. The case of the ordinary superpotential is a special case of this with $g=0, h=1$.

\subsection{Dual Type IIB perspective}

We have already given one dual type IIB theory related to our type IIA geometry, and that is given by the mirror symmetry we have been considering. However there is another type IIB dual description which is also rather useful.

Consider M-theory on a non-compact Calabi-Yau $X$ compactified to 5 dimensions, which admits a $T^{2}$ action, possibly with fixed points. We can use the duality of M-theory on $T^{2}$ with type IIB on $S^{1}$ 23 to give a dual type IIB description for 
this class of Calabi-Yau manifolds. Note that the complex structure of the $T^{2}$ gets mapped to the coupling constant of type IIB. The non-compact Calabi-Yau manifolds we have been considering do admit $T^{2}$ actions and in this way they can be mapped to an equivalent type IIB theory. This in fact has been done in [24] where it was shown that this class of CY gets mapped to type IIB propagating on a web of $(p, q) 5$-branes considered in [25]. In this picture the 5 -branes fill the 5 dimensional space time and extend along one direction in the internal space, identified with various edges of the toric diagram. The choice of $(p, q) 5$-branes encodes the $(p, q)$ cycle of $T^{2}$ shrinking over the corresponding edge. The 5-branes are stretched along straight lines ending on one another and making very specific angles dictated by the supersymmetry requirement (balancing of the tensions) depending on the value of the type IIB coupling constant $\tau$. In particular each $(p, q)$ fivebrane is stretched along 1 dimensional line segments on a 2-plane which is parallel to the complex vector given by $p+q \tau$. An example of a configuration involving a D5 brane, NS 5 brane and a (1,1) 5 -brane is depicted in Fig. 6.

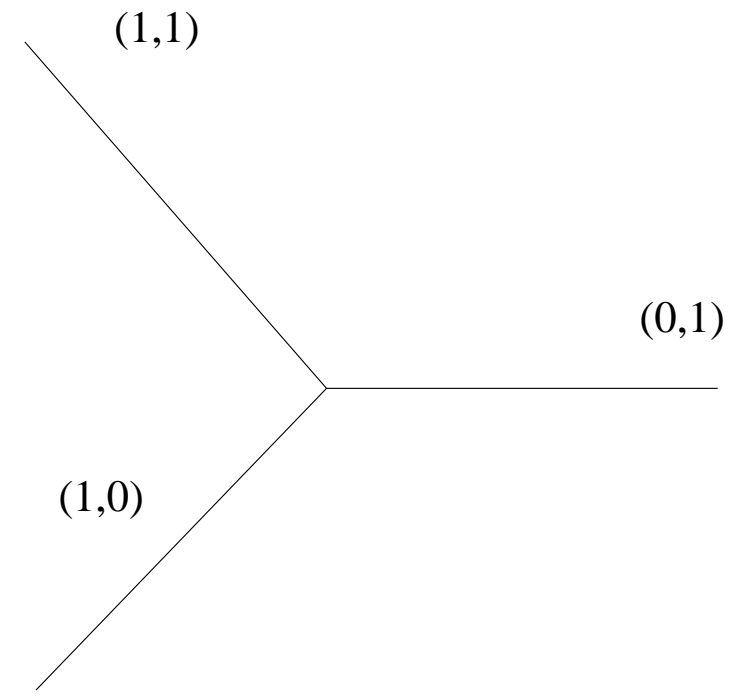

Fig.6 $A(p, q)$ web of 5-branes which is dual to $M$-theory on $\mathbf{C}^{\mathbf{3}}$. This web is a junction involving a D5 brane which has $(p, q)=(1,0)$, an NS5 brane which is $(0,1)$ and a $(1,1)$ brane.

Now we recall from the previous discussion that to get the $G_{2}$ holonomy manifold we need to consider an extra $S^{1}$ which is fibered over the corresponding CY. In other words we are now exchanging the '5-th' circle with the '11-th' circle. So we consider 
going down to 4 dimensions on a circle which is varying in size depending on the point in Calabi-Yau. In particular the circle (i.e. the one corresponding to the 5 -th dimension) vanishes over a 2-dimensional subspace of 5-dimensional geometry of type IIB (it vanishes along the radial direction of the Lagrangian submanifold on the base of the toric geometry as well as on the $S^{1}$ which is dual to the $T^{2}$ of M-theory). Indeed it corresponds to putting the IIB 5-brane web in a background of ALF geometry dictated by the location of the Lagrangian submanifold in the base times $S^{1}$, and varying the geometry and splitting the ALF geometry to two halves, as shown in Fig.2. In this picture the worldsheet disk instantons of type IIA get mapped to $(p, q)$ Euclidean worldsheet instantons, wrapping the 5-th circle and ending on the 5-branes. In particular if we follow the map of the Euclidean instanton to this geometry it is the other disk realization of $S^{3}$ (see Fig.7) . 2

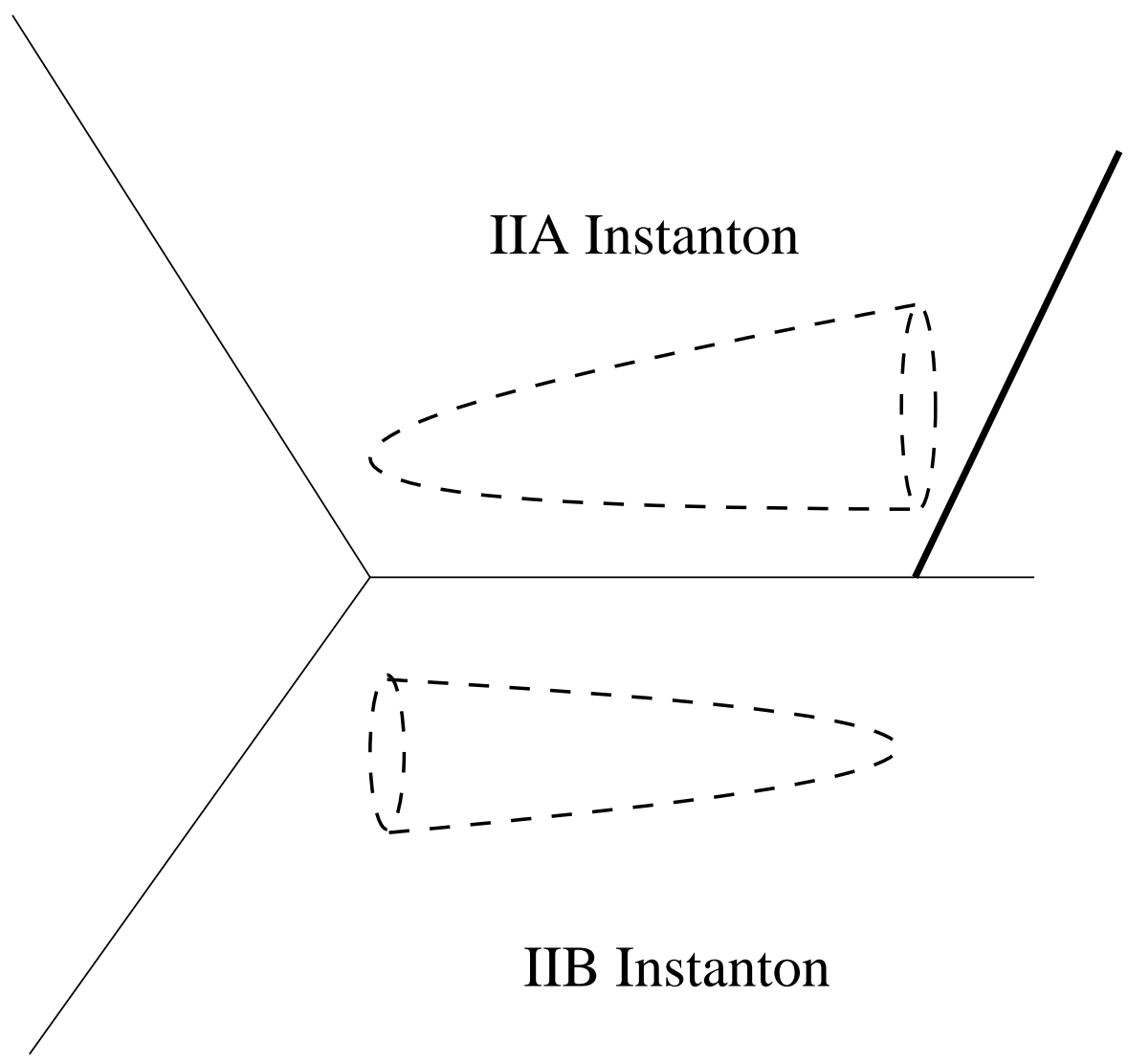

2 Note that a D6 branes wrapped around $S^{3}$ is realized in type IIB as an $N S 5$-brane in the $x$ direction, a $D 5$ brane in the $y$ direction separated in the $z$ direction, and where the 5 -th circle vanishes along the interval in the $z$ direction joining the two branes. 
Fig.7 The M2 brane instanton with a topology of $S^{3}$ wrapping over a 3-cycle of a local $G_{2}$ manifold gets mapped to two alternative disk projections of $S^{3}$, depending on which duality we use. In one case we get the description involving a type IIA string theory on a $C Y$ with D-brane wrapped over Lagrangian submanifold and in the other we get a web of IIB 5-branes in the presence of ALF like geometries.

\section{Choice of Flat Coordinates}

In this section we consider the map between the moduli of the brane between the A- and B-model.

As discussed in section 2, the moduli of the D-branes in the A-model, are labeled by $c$ which measures the size of the disk instanton ending on the Lagrangian submanifold. In the quantum theory $c$ gets complexified by the choice of the Wilson line on the brane, and get mapped to the choice of a complex point on the mirror type IIB geometry. The choice is characterized by the choice of a point on a Riemann surface $F(u, v)=0$, which we choose to be our ' $u$ ' variable. However it could be that the 'size' of the disk instanton, receives quantum corrections, and this, as we will now discuss is relevant for finding the natural ("flat") coordinates parameterizing the moduli space of Lagrangian D-branes.

First we have to discuss what we mean by the "natural" choice of coordinate for the A-model. This is motivated by the integrality structure of the A-model expansion parameter. There is a special choice of coordinates [4] on the moduli space of D-branes in terms of which $A$-model disk partition function has integer expansion (2.11), and this is the coordinate which measures the tension of the $D 4$ brane domain walls. There is no reason to expect this to agree with the classical size of the disk that the B-model coordinate measures, and in general the two are not the same, as we will discuss below. This is what we take as the natural coordinates on the A-model side.

The B-model and the A-model are equivalent theories, and this dictates the corresponding flat coordinate on the $B$ model moduli space. This is the tension of the domain-wall D-brane which is mirror brane to the D4 brane of the A-model.

The D4 brane wrapping a minimal disk $D$ is magnetically charged under the gauge field on the D6 brane. Consider the domain wall which in the $R^{3,1}$ is at a point in $x_{3}$ and fills the rest of the spacetime. The Bianchi identity for the gauge 
field-strength $F$ on $L$, modified by the presence of the D4 brane, says that if the $B$ is the cycle Poincare dual to the boundary of the disk $\partial D \subset L$. Recall that our brane $L$ has the topology of $\mathbf{C} \times S^{1}$, so $B$ can be identified with $\mathbf{C}$. Then the charge $n$ of the domain wall is measured by

$$
2 \pi n=\int_{B} F\left(x_{3}=\infty\right)-F\left(x_{3}=-\infty\right)=\int_{\partial B} A\left(x_{3}=\infty\right)-A\left(x_{3}=-\infty\right)
$$

Recall that $\operatorname{Im} u$ and $\operatorname{Im} v$ map to the one forms related to the $S^{1} \times S^{1}$ cycles of the Lagrangian geometry, viewed as a cone over $T^{2}$. Thus $\operatorname{Im}(u)$ is the mirror of the Wilson-line $\int_{\partial D} A$, and the Wilson-line around the dual $S^{1}=\partial B$ is identified with $\operatorname{Im}(v)$. Thus we find that the $v$ jumps over the mirror domain wall by

$$
v \rightarrow v+2 \pi i n
$$

The case of $n=1$ is depicted in Fig. 8 .
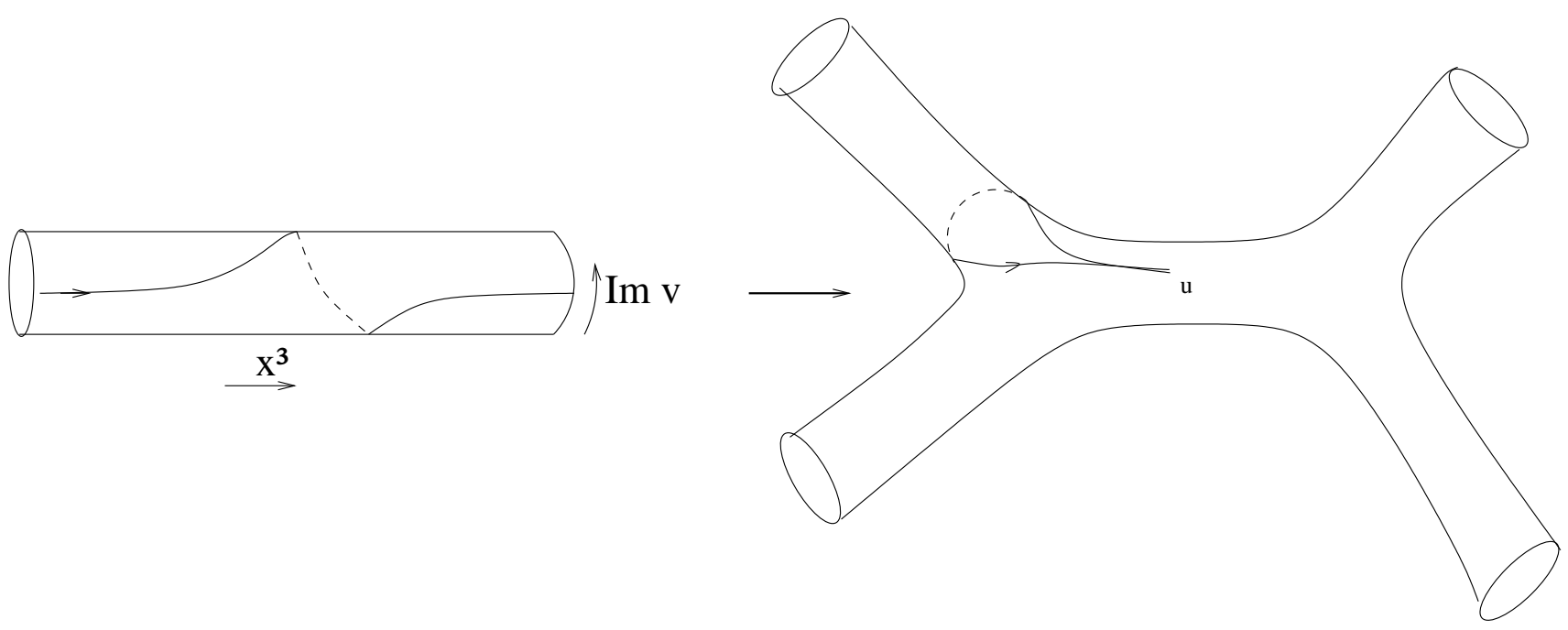

Fig.8 The D4 brane ending on the D6 brane is mirrored to a domain wall in type IIB where $v$ shifts by $2 \pi i$ across it. This projects to a closed cycle on the Riemann surface $P(u, v)=0$.

This allows us to find the tension of the mirror domain wall as discussed in [6]. The BPS tension of the domain wall is given by $\Delta W$ the difference of the superpotentials on the two sides of the domain wall. Since $W=\frac{1}{2 \pi i} \int_{u_{*}}^{u} v d u$, the tension of the BPS domain wall is simply the integral $\frac{1}{2 \pi i} \int_{C_{u}} v d u$ where $C_{u}$ denotes 
the appropriate cycle shifting $v \rightarrow v+2 \pi i$, beginning and ending on a given $u$. We thus define the flat coordinate

$$
\hat{u}(u, \vec{t})=\frac{1}{2 \pi i} \int_{C_{u}} v d u
$$

To summarize, we predict that the disk partition function (2.10), expanded in terms of $\hat{u}=\hat{u}(u, \vec{t})$ and the corresponding closed string counterpart $\hat{t}(t)$ - has integral expansion (2.11) the coefficients of which count the "net number" of D4 brane domain walls ending on the Lagrangian submanifold $L$ (for a more precise definition see [4] 26]).

It is not hard to see that $u$ and $\hat{u}$ as defined above agree at the classical level and differ by instanton generated corrections. In the large radius limit, the local A-model geometry in the neighborhood of the disk $D$ is just $\mathbf{C}^{\mathbf{3}}$ - all toric vertices other than the one supporting $D$ go away to infinity. In this limit, the equation of the mirror simply becomes $P(u, v) \rightarrow e^{u}+e^{v}+1$ so

$$
\hat{u}=\frac{1}{2 \pi i} \int_{C_{u}} v(u) d u \rightarrow \frac{1}{2 \pi i} \int_{C_{u}}\left[\log \left(1+e^{u}\right)+i \pi\right] d u
$$

This has a branch point in the $u$-plane around which $v$ has monodromy $v \rightarrow v+2 \pi i$. The contour $C_{u}$ receives contribution only from difference of values of $v$ on the two sides of the cut, and thus for a single domain-wall $\hat{u}=u+i \pi$.

Away from the classical limit we can have subleading corrections to the above relation that can in principle be subleading in $e^{-t}$ and in $e^{-u}$. But we will now argue that it is of the form

$$
\hat{u}=u+\text { const. }+O\left(e^{-t}\right)
$$

In other words, we show that $\delta \hat{u} / \delta u=1$ is exact even away from the classical limit. This in fact is obvious from the definition of (4.1) because as we change $u$, the change in (4.1) can be computed from the beginning and the end of the path. But the integrands are the same except for the shift of $v$ by $2 \pi i$, and therefore the difference is given by $\delta \hat{u}=\delta \int d u=\delta u$. We have thus shown that $\hat{u}$ differs from $u$ by closed string instanton corrections only. Another way to see this is to note that

$$
\hat{u}=\frac{1}{2 \pi i} \int_{C_{u}} v d u=\frac{1}{2 \pi i} \Delta(u v)-\frac{1}{2 \pi i} \int_{C_{u}} u d v
$$


Noting that $\Delta(u v)=2 \pi i u$ due to the shift in $v$, and using the fact that $u$ is not shifting and that $d v$ is well defined, we deduce that the $-\frac{1}{2 \pi i} \int_{C_{u}} u d v=\Delta$ is independent of $u$, by the deformation of the contour $C_{u}$, and only depends on the class of the contour $C$. It thus depends only on the bulk moduli.

A cautionary remark is in order. We have talked about cycle $C_{u}$ on the B-model side as a cycle on the Riemann surface $F(u, v)=0$. In general the cycles on the Riemann surface $F(u, v)=0$ can be divided to those that lift to cycles where $u$ and $v$ come back to the original values, or those that shift by an integer multiples of $2 \pi i$. The cycles that come back to themselves without any shifts in $u$ and $v$ correspond to closed 3-cycles in the underlying CY. Integration of $v d u$ over those cycles correspond to computation of electric and magnetic BPS masses for the underlying $N=2$ theory in 4 dimensions (and are relevant for the computation of the "flat" coordinate for the bulk field $\hat{t}(t))$. However, the cycles whose $u$ or $v$ values shift by an integer multiple of $2 \pi i$ do not give rise to closed 3-cycles in the CY (as the CY in question does have non-trivial cycles corresponding to shifting $u$ or $v$ by integer multiples of $2 \pi i$ ). Nevertheless as we discussed above such cycles are important for finding the natural coordinates in the context of D-branes.

Note that closed string periods which determine $\hat{t}$ can also be expressed in terms of linear combinations of periods where $u$ 's and $v$ 's shift. Thus computing periods where $u$ and $v$ shift are the fundamental quantities to compute. We will discuss these in the context of examples in section 6 .

Just as we have defined $\hat{u}$ as the quantum corrected tension of domain wall, we can define $\hat{v}$ at the quantum corrected tension of the domain wall associated with shifting $u \rightarrow u+2 \pi i$. Note that in the derivation of the superpotential [6] $u, v$ are conjugate fields of the holomorphic Chern-Simons field. Thus replacing $u \rightarrow \hat{u}$ will require 3 replacing $v$ by the quantum corrected conjugate field $\hat{v}$ and so the equation

3 To see this from the target space viewpoint it is natural to consider the $1+1$ realization of this theory as D4 brane wrapped over the Lagrangian submanifold. Then, as discussed in 《4 the disk amplitude computes $S=\int d^{2} x d^{2} \theta(d W / d \Sigma) \Sigma$ for the $U(1)$ gauge theory in $1+1$ dimension where $\Sigma$ is the twisted chiral gauge field strength multiplet, whose bottom component is $\hat{u}$. In this formulation the domain wall associated with shifting of $\Sigma \rightarrow \Sigma+2 \pi i$ is realized by $u \rightarrow u+2 \pi i$, whose BPS mass we have denoted by $\hat{v}$. From $S$, the change in the value of the superpotential under shifting $\Sigma$ is given by $d W / d \hat{u}$ which leads to the statement that $d W / d \hat{u}=\hat{v}$. This provides an alternative, and more physical derivation of the main formula we use for computation of $W$. 
satisfied by the superpotential changes to

$$
\frac{\partial W}{\partial u}=v \rightarrow \frac{\partial W}{\partial \hat{u}}=\hat{v}
$$

which is the equation we will use in section 6 to compute $W$.

\section{Quantum Ambiguity for Lagrangian Submanifold}

We have seen that the choice of flat coordinates naturally adapted to the Amodel Lagrangian D-branes are fixed up to an integer choice. In particular we found that if $u, v$ are complex coordinates satisfying $P(u, v)=0$ and if the brane is denoted in the classical limit by $v=0$ and $u$ classically measures the size of the disk instanton, then we can consider a new $u$ given by

$$
u \rightarrow u+n v
$$

for any $n$, which classically still corresponds to the disk instanton action. In this section we explain why fixing the arbitrary choice is indeed needed for a quantum definition of the A-model Lagrangian D-brane. In particular specifying the A-model Lagrangian D-brane just by specifying it as a classical subspace of the CY does not uniquely fix the quantum theory, given by string perturbation theory. The choice of $n$ reflects choices to be made in the quantum theory, which has no classical counterpart. In this section we show how this works in two different ways: First we map this ambiguity to an UV Chern-Simons ambiguity related to framing of the Wilson Loop observables. Secondly we relate it to the choice of the Calabi-Yau geometry at infinity, and for this we use the type IIB 5-brane web dual, discussed in section 3.

\subsection{Framing Choices for the Knot}

To see how this works it is simplest to consider the case where the D-brane topological amplitudes were computed using the observables of Chern-Simons theory [4] [27] [26]. These were obtained by considering expectation values for Wilson loop observables in the large $N$ Chern-Simons theory, in the context of the large $N$ duality of Chern-Simons/closed topological strings proposed in [28]. Let us briefly recall this setup. 
Consider $S U(N)$ Chern-Simons theory on $S^{3}$. As was shown in [29] if we consider topological A-model on the conifold, which has the same symplectic structure as $T^{*} S^{3}$, and consider wrapping $N$ D3 branes on $S^{3}$, the open string field theory living on the D3 brane is $S U(N)$ Chern-Simons theory where the level of Chern-Simons theory (up to a shift by $N$, i.e., $g_{s}=2 \pi i /(k+N)$ ) is identified with the inverse of string coupling constant. The large $N$ duality proposed in [28] states that this topological string theory is equivalent to topological strings propagating on the noncompact CY 3-fold $O(-1) \oplus O(-1) \rightarrow \mathbf{P}^{1}$, which is the resolution of the conifold, where the complexified Kähler class on $\mathbf{P}^{1}$ has size $t=N g_{s}$. In [4] it was shown how to use this duality to compute Wilson loop observables. The idea is that for every knot $\gamma \subset S^{3}$ one considers a non-compact Lagrangian submanifold $L_{\gamma} \subset T^{*} S^{3}$ such that $L_{\gamma} \cap S^{3}=\gamma$. We wrap $M$ D3 branes over $L_{\gamma}$ which gives rise to an $S U(M)$ Chern-Simons gauge theory on $L_{\gamma}$. In addition bi-fundamental fields on $\gamma$ transforming as $(N, \bar{M})$, arise from open strings with one end on the D-branes wrapped over $S^{3}$ and with the other end on D-branes wrapped over $L_{\gamma}$. Integrating out these fields give rise to the insertion of

$$
\left\langle\exp \left(\sum_{n} \frac{\operatorname{tr}^{\mathrm{n}} \operatorname{tr} \mathrm{V}^{\mathrm{n}}}{\mathrm{n}}\right)\right\rangle
$$

where $U$ and $V$ denote the holonomies of the $S U(N)$ and $S U(M)$ gauge groups around $\gamma$ respectively. Considering the $S U(M)$ gauge theory as a spectator we can compute the correlations of the $S U(N)$ Chern-Simons theory and obtain

$$
\left\langle\exp \left(\sum_{n} \frac{\operatorname{tr}^{\mathrm{n}} \operatorname{trV}^{\mathrm{n}}}{\mathrm{n}}\right)\right\rangle=\exp \left(-F\left(V, t, g_{s}\right)\right)
$$

It was shown in [4] that the right-hand side can be interpreted as the topological string amplitude in the large $N$ gravitational dual, where the $N$ D-branes have disappeared and replaced by $S^{2}$. In this dual geometry the $M$ non-compact D-branes are left-over and wrapped over some Lagrangian submanifold in $O(-1) \oplus O(-1) \rightarrow \mathbf{P}^{1}$. This Lagrangian submanifold was constructed for the case of the unknot explicitly in [4] and extended to algebraic knots in [26] (this latter construction has been recently generalized to all knots [30] $)$. Moreover $F\left(V, t, g_{s}\right)$ denotes the topological string amplitude in the presence of the $M$ D-branes wrapped over some particular Lagrangian submanifold in $O(-1) \oplus O(-1) \rightarrow \mathbf{P}^{1}$ with a non-trivial $S^{1}$ cycle. Note that a term in 
$F\left(V, t, g_{s}\right)$ of the form $\prod_{i=1}^{b} \operatorname{trV}^{\mathrm{k}_{\mathrm{i}}}$ comes from a worldsheet with $b$ boundaries where the $i$-th boundary of the worldsheet wraps the $S^{1}$ of the Lagrangian submanifold $k_{i}$ times.

The left-hand side of (5.1) is computable by the methods initiated in [8] and in this way gives us a way to compute the open string topological amplitudes for this class of D-branes. Note in particular that the disk amplitude corresponds to the $1 / g_{s}$ term in $F\left(V, t, g_{s}\right)$. A particular case of the brane we have considered in $O(-1) \oplus O(-1) \rightarrow \mathbf{P}^{1}$ corresponds to the unknot. This is depicted in the toric Fig. 2.

The match between the computation in this case, using mirror symmetry and the result expected from Chern-Simons theory was demonstrated in [6]. However as we have discussed here the disk amplitudes have an integer ambiguity, when we use mirror symmetry for their computation. Thus apparently the right hand side of (5.1) is defined once we pick an integer, related to the boundary conditions at infinity on the B-brane in the type IIB mirror. If the right hand side of (5.1) is ambiguous, then so should the left hand. In fact the computation of Wilson loop observables also has an ambiguity given by an integer! In particular we have to choose a framing on the knot $\gamma$ to make the computation well defined in the quantum theory [8]. A framing, is the choice of a normal vector field on the knot $\gamma$, which is non-vanishing everywhere on the knot. Note that if we are given a framing of the knot, any other topologically distinct framing is parameterized by an integer, given by the class of the map $S^{1} \rightarrow S^{1}$, where the domain $S^{1}$ parameterizes $\gamma$ and the range denotes the relative choice of the framing which is classified by the direction of the vector field on the normal plane to the direction along the knot. The framing of the knot enters the gauge theory computation by resolving UV divergencies of the Chern-Simons theory in the presence of Wilson loops. It arises when we take the Greens function for the gauge field coming from the same point on the knot. The framing of the knot allows a point splitting definition of the Greens function.

We have thus seen that both sides of (5.1) have a quantum ambiguity that can be resolved by a choice of an integer. On the left hand side the ambiguity arises from the UV. On the right hand side the ambiguity arises from the IR (i.e. boundary conditions on the brane at infinity). We have checked that the two ambiguities match for the case of the unknot, by comparing the disk amplitudes on both sides (using CS computation of the framing dependence of the knot on the left and comparing 
it with the mirror symmetry computation of the knot on the right). Some aspects of this computation is presented in the appendix A. The computation of the disk amplitude for this case using mirror symmetry is presented in section 6 .

Here let us discuss further how this match arises. Consider the disk amplitude at large $N$ corresponding to a given knot. In the gauge theory side the computation arises from open string diagrams of a planar diagram with the outer hole on the Lagrangian submanifold $L_{\gamma}$, and rest of the holes ending on D-branes wrapping the $S^{3}$, as shown in Fig.9. In the large $N$ limit, the interior holes get "filled" and we get the topology of the disk.
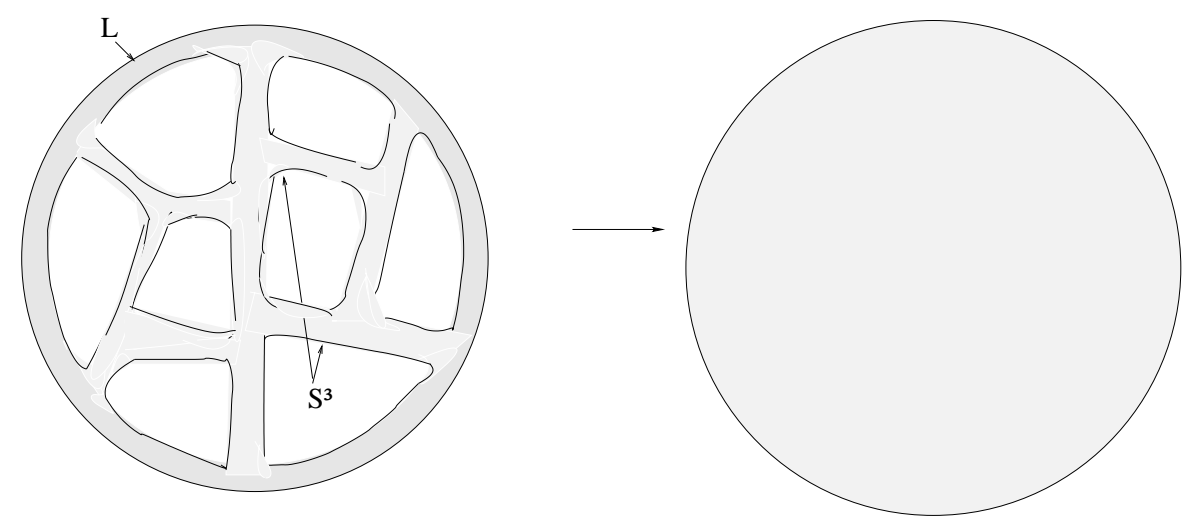

Fig.9 The Wilson loop observables arise from worldsheet diagrams where some boundaries end on $L_{\gamma}$ and $N$ branes wrapping $S^{3}$. In the large $N$ limit the holes ending on $S^{3}$ get "filled" and we end up with a Riemann surface which has only the boundaries associated with $L_{\gamma}$. In the above figure the outer hole is the only one ending on $L_{\gamma}$. All of the interior holes end on $S^{3}$ and disappear in the large $N$ limit, leaving us with a disk.

Now consider where the UV divergencies of the gauge theory would arise. They would arise from Feynman diagrams where the Schwinger parameter for the gauge field goes to zero-an example of this is depicted in Fig.10a. Note that the two end points of the short propagator will be mapped to the same point on the knot $\gamma$ in the limit of zero length propagator. In the large $N$ limit, where the disk gets filled these get mapped to configurations such as that shown in Fig.10b. In this dual description by a conformal transformation the worldsheet can be viewed as that depicted in Fig.10c. In other words we have in the dual channel a long schwinger time, of an open string ending on $L_{\gamma}$. This means that the issue of ambiguity is mapped to an 
IR behaviour of fields living on $L_{\gamma}$, and that is exactly where we found the ambiguity in the computation of the superpotential in the context of mirror symmetry.

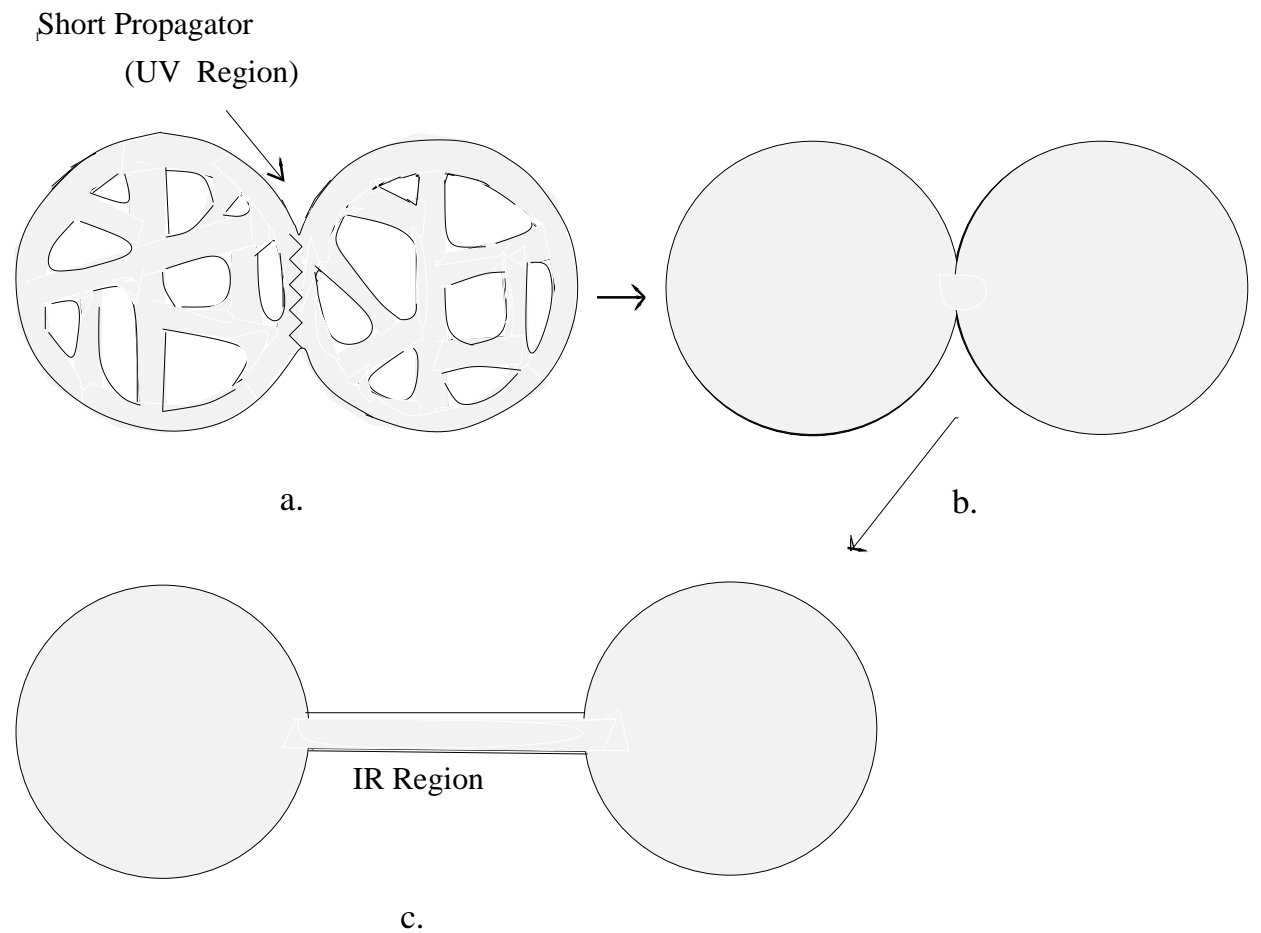

Fig.10 The Wilson loop observable has UV divergencies which need to be regulated, coming from points along the knot where the gauge boson propagator is of zero size (a). In the large $N$ limit these map to disks touching at a point (b), which can be viewed via a conformal transformation as a long propagator (c). Note that the boundaries of the long propagator are on the Lagrangian submanifold $L_{\gamma}$ (or more precisely its large $N$ dual) and correspond to open string propagating on it. Thus the UV framing choice of CS gets mapped to the choice of large distance (IR) physics of modes on the brane.

Other examples of the large $N$ limit of UV regions for the computation of the Wilson loop observable along the knot get mapped to disks shown in Fig.11, and look like branched trees of disks. 


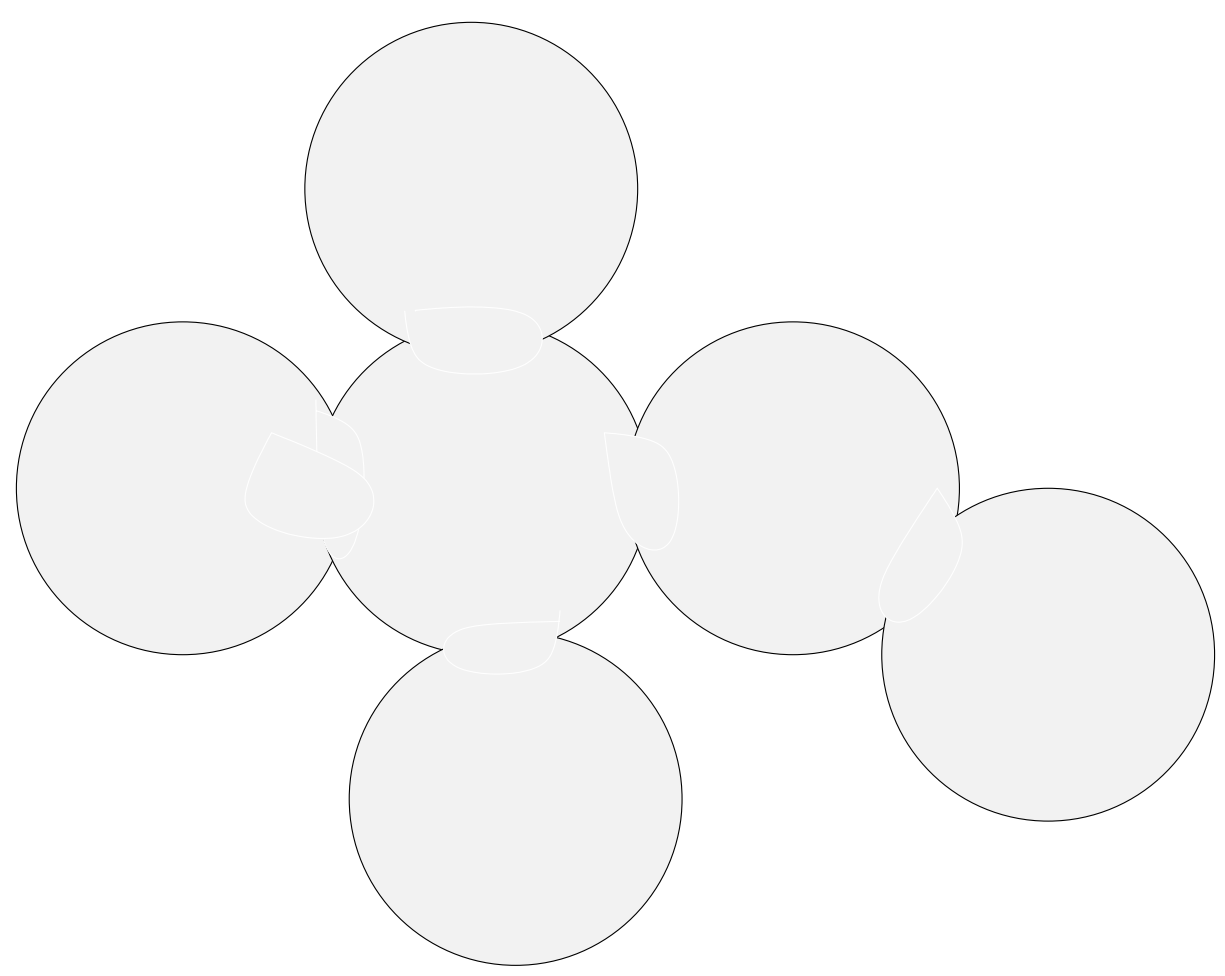

Fig.11 Other examples of large $N$ limit of $U V$ divergencies of Wilson loop observables.

\subsection{Calabi-Yau Geometry, 5-brane Perspective and the Integral Ambiguity}

As discussed in section 3 we have dual descriptions of type IIA geometry with D6 branes wrapped over Lagrangian submanifolds in terms of M-theory on $G_{2}$ holonomy manifolds (viewed as circle fibration over CY manifolds) or in terms of type IIB web of 5 branes in an ALF-like background in $R^{6}$. To be precise, the M-theory on $T^{2} / \operatorname{type}$ IIB on $S^{1}$ duality relates the coupling constant of type IIB to the complex structure of the $T^{2}$. Fibering this duality gives rise to the duality just mentioned. However, in the type IIB picture we typically fix the type IIB coupling at infinity. This means that, by this chain of duality, the $G_{2}$ holonomy manifold is a circle fibration over the CY where the complex structure of the $T^{2}$ fibration of $\mathrm{CY}$ is fixed at infinity. Turning this around, this duality predicts the existence of particular class of CY and $G_{2}$ holonomy metrics with particular behaviour of the metric at infinity (we can also fix the area of $T^{2}$ at infinity as that gets mapped to the inverse of the radius of type IIB $S^{1}$ ). We now argue that the choice of the complex structure of $T^{2}$, or equivalently type IIB coupling constant at infinity changes the number of Euclidean M2 branes, exactly as is expected by the ambiguity. Instead of being general we simply illustrate 
this idea in the context of a simple example, namely the $\mathbf{C}^{3}$ geometry discussed before and represented by Fig.6, consisting of NS 5-brane $(0,1)$ and D5-brane $(1,0)$ and the $(1,1)$ 5-brane. We already discussed that M2 brane instantons get mapped to worldsheet disk instantons consisting of the $(p, q)$ string shown in Fig.7 times an extra circle (the '5-th' circle) which vanishes at the position of the ALF space. However now consider changing the coupling constant. Then the angles between the 5-branes will be changed. Beyond some critical angles we can get new worldsheet instantons. Basically we can consider the web of strings and the only data that we need to take into account is that a $\operatorname{BPS}(p, q)$ string can end only on a $(p, q)$-brane (and perpendicularly). This web of strings however, can end on the projection of the ALF space on the web at any angle since at that point now the '5-th' circle is shrinking (again in a perpendicular fashion). To be concrete let us consider the limiting choices of the type IIB coupling constant given by $\tau \rightarrow-1 / n$. This can be obtained from $\tau \rightarrow i \infty$ by the modular transformation $\frac{-1}{\tau} \rightarrow \frac{-1}{\tau}+n$. Said differently we can take the weak coupling limit $\tau \rightarrow i \infty$ by considering the inverse modular transformation where we consider the 5 -brane web given by NS fivebrane $(0,1)$ and the fivebranes $(1, n)$ and $(1, n+1)$. It is easy to see that this geometry of webs has an $n \rightarrow-(n+1)$ symmetry, when we reverse the direction of the handedness on the D5 brane. Thus, the counting of the worldsheet instantons here will exhibit this symmetry. This is exactly the symmetry we will find for the ambiguity of the mirror to $\mathbf{C}^{3}$, as discussed in section 6 . Moreover it is easy to see that except for $n=0,-1$ where there is exactly one choice of disk instanton, for all other $n$ 's we get a large number of disk instantons, allowed by the geometry of the 5-branes, as shown in Fig.12. This is also in line with the result we find from mirror symmetry in section 6. Thus as the geometry of Calabi-Yau changes, we get jumps in the number of instantons as predicted by this picture. 

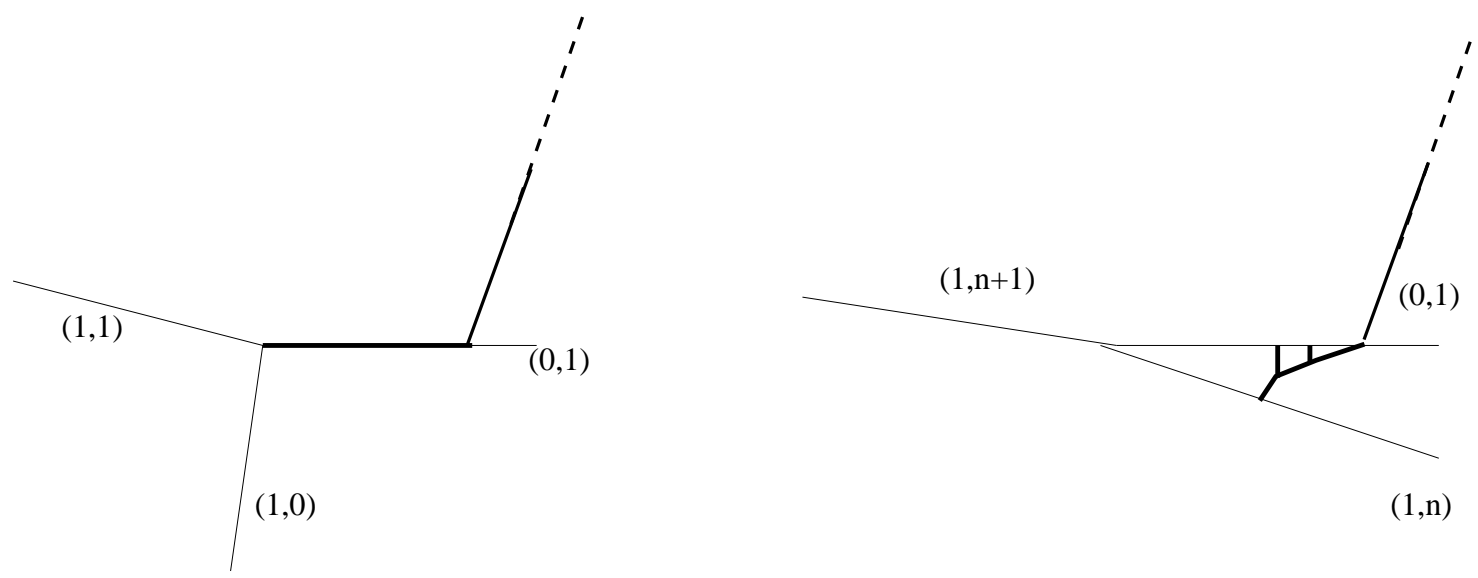

$(1, \mathrm{n})$

Fig. 12 In the weak coupling limit, we consider an $N S(0,1)$ 5-brane with an ALF like space ending on it. If this is connected to $(1,0)$ and $(1,1) 5$-branes in a junction, the only instantons allowed involve the worldsheet instanton stretched along the interval shown on the left-hand side of the figure above, times the 5-th circle which shrinks at the attachment point of the ALF space. There is no other instanton allowed in this geometry. However if NS $(0,1) 5$-brane is attached to $(1, n)$ and $(1, n+1) 5$-branes the geometry of the intersection dramatically changes (as long as $n \neq 0,-1$ ) and now we can have many more allowed configurations for the worldsheet $(p, q)$ instantons. This is depicted on the right hand side of the above figure.

It is natural to ask what IR aspect of the type IIA CY geometry with the brane, this choice of $\tau$ is reflected in. One's natural guess is that the normalizability of the 1-form on the brane represented by the Wilson line vev, which represents the mode corresponding to moving the brane, is the relevant issue. If this is the case the possible choices of metrics for which a particular 1-form is normalizable will have to be classified by the integer ambiguity $n$ which in turn is related to the choice of the CY metric with fixed $\tau$ at infinity, labeled by $n$. Similarly in the M-theory lift this would be related to the possible choices of normalizable deformations of the $G_{2}$ holonomy metric. It would be interesting to study these issues further.

\section{Examples}

In this section we will consider a number of examples which illustrate the general discussion we have presented for the computation of disk amplitudes for D-branes wrapping Lagrangian submanifold of non-compact CY 3-folds. The examples include 
considerations of Lagrangian branes in $\mathbf{C}^{3}, \mathbf{P}^{1} \times \mathbf{P}^{1}, \mathbf{P}^{2}$ and its blow up at a point $\mathbf{F}_{1}$. In these examples we consider inequivalent configurations of Lagrangian branes. For the case of $\mathbf{C}^{3}$ we exhibit the dependence of the topological string computations on the integer ambiguity we discussed earlier. For all of these cases we find the predicted integrality properties of the disk amplitudes. We also consider different Lagrangian branes (for example ending on the "internal" or "external" edges of the toric diagrams).

As discussed in section 4, a non-trivial part of the story involves finding the flat coordinates for the open string variables. As discussed there we find the flat coordinates to be given by

$$
\hat{u}=u+\Delta\left(t_{i}\right)
$$

where $\Delta\left(t_{i}\right)$ (up to an addition of a constant) is an exponentially suppressed function of Kähler moduli of the Calabi-Yau $\left(O\left(e^{-t_{i}}\right)\right)$, and $\Delta$ depends on the choice of the brane. We compute $\Delta$ using the methods discussed in section 4 for all the examples.

Note that using $\partial_{\hat{u}} W=\hat{v}(\hat{u})$ and $(2.11)$ we have

$$
\partial_{\hat{u}} W=\hat{v}(\hat{u})=-\sum_{k} k N_{k, \vec{m}} \log (1-\exp [k \hat{u}-\vec{m} \cdot \overrightarrow{\hat{t}}])
$$

and so by solving for $\hat{v}$ in terms of $\hat{u}$ (from $P(u, v)=0$ and the correction to $u, v \rightarrow \hat{u}, \hat{v}$ due to mirror map) we find a prediction of an expansion in terms of integers $N_{k, \vec{m}}$. We verify these highly non-trivial integrality predictions in the examples below which we now turn to.

\subsection{Almost $\mathbf{C}^{3}$}

We consider the simplest Calabi-Yau $X=\mathbf{C}^{\mathbf{3}}$. It is described by just three chiral fields $X^{i}$ and no gauge group. The special Lagrangian D-brane $L$ we are interested in is given by $q_{1}=(1,0,-1)$ and $q_{2}=(0,1,-1)$ :

$$
\left|X^{1}\right|^{2}-\left|X^{3}\right|^{2}=c_{1} \quad\left|X^{2}\right|^{2}-\left|X^{3}\right|^{2}=0
$$

4 The integrality requirement is highly non-trivial. Originally, we experimentally found the highly non-trivial flat coordinates only by requiring the integrality of the domain wall degeneracies. Later we showed that they agree with the BPS tension of domain walls, which is the definition used in this section. 
This geometry can be regarded as a local approximation, in the limit all radii are large, to a more involved geometry it is embedded in, for example the one discussed in section 2. The value of $c_{1}$ on the brane and the Wilson-line around the one finite circle on $L$ form one complex modulus $u$ of the A-brane, which measures the classical BPS tension of the D4 brane domain wall.

The mirror manifold can be written as

$$
x z=P(u, v)=e^{u}+e^{v}+1
$$

where we have set $Y^{3}$ to zero, and $Y^{1}=u, Y^{2}=v$.

Equations (2.3) fix the classical limit of the brane

$$
c^{1}=\operatorname{Re}(u)
$$

so the transverse coordinate to the B-brane is $u$. In this limit, $v$ is zero on the brane corresponding to the vanishing of the classical superpotential.

The $\mathrm{SL}(2, \mathbb{Z})$ group of reparametrizations acts on $(6.1)$ by linear transformations $\left(\begin{array}{l}\hat{u}^{\prime} \\ \hat{v}^{\prime}\end{array}\right)=\left(\begin{array}{ll}a & b \\ c & d\end{array}\right)\left(\begin{array}{l}\hat{u} \\ \hat{v}\end{array}\right)$ that leave the holomorphic $(3,0)$ form $\Omega=\frac{\mathrm{d} z}{z} \mathrm{~d} u \mathrm{~d} v$ invariant. The subgroup of $\operatorname{SL}(2, \mathbb{Z})$ that preserves the classical limit above consists of transformations $\left(\begin{array}{ll}a & b \\ c & d\end{array}\right)=\left(\begin{array}{ll}1 & p \\ 0 & 1\end{array}\right)$. These transformations result in a family of parameterizations of the mirror geometry

$$
x z=P_{p}(u, v)=e^{u+p v}+e^{v}+1,
$$

and a family of superpotentials obtained by solving the order $p$ polynomial in $e^{v}$,

$$
W_{p}(u)=\int v_{p}(u) d u
$$

To compute the numbers of disk domain walls we need to know the flat coordinates.

For $p=0$ we have computed in section 4 the BPS tension of the D-brane in $u$ phase and also the $v$-phase, by trivial relabeling, to be $\hat{u}_{p=0}=u+i \pi, \hat{v}_{p=0}=v+i \pi$. The knowledge of these suffices to find the BPS tensions for a different choice of framing $p$.

The $\mathrm{SL}(2, \mathbb{Z})$ transformations $u \rightarrow u+p v, v \rightarrow v$ act on the basis of one-cycles on the Riemann surface that are associated to periodicity of $\operatorname{Im}(u)$ and $\operatorname{Im}(v)$. Since $\Delta_{u}$ and $\Delta_{v}$ which provide the quantum corrections to $u$ and $v$,

$$
\hat{u}=u+\Delta_{u}, \quad \hat{v}=v+\Delta_{v}
$$


are obtained by evaluating periods of the one-form $\lambda=v d u$ over these, changing the basis of one-cycles by an $\mathrm{SL}(2, \mathbb{Z})$ transformation to $u^{\prime}=a u+b v$ and $v^{\prime}=c u+d v$ acts in an analogous way on the periods i.e.

$$
\Delta_{u}^{\prime}=a \Delta_{u}+b \Delta_{v}
$$

Thus, we find that

$$
\hat{u}_{p}=u+(p+1) i \pi, \quad \hat{v}_{p}=v+i \pi
$$

The equation

$$
P_{p}(\hat{u}, \hat{v})=0=1-e^{\hat{u}+p \hat{v}}-e^{\hat{v}}
$$

is solved iteratively using the ansatz $e^{\hat{v}}=\sum_{k=0}^{\infty} a_{k} e^{k \hat{u}}$. Using $\left(\sum_{k=0}^{\infty} a_{k} x^{k}\right)^{p}=$ $\sum_{m=0}^{\infty} c_{m} x^{m}$, where $a_{0}=c_{0}=1$ and $c_{m}=\frac{1}{m} \sum_{k=1}^{m}(k p-m+k) a_{k} c_{m-k}$ we get immediately a recursive formula $a_{m}=\frac{1}{m-1} \sum_{m=1}^{m-1}(k p-m+k+1) a_{k} a_{m-k}$ for the coefficients. This can be summed using Stirlings coefficients of the first kind (see e.g. [31], which are defined by $x(x-1)(x-2) \cdot \ldots \cdot(x-n+1)=\sum_{m=0}^{n} S_{n}^{(m)} x^{m}$. Using the relation $\left(\begin{array}{c}m \\ r\end{array}\right) S_{n}^{(m)}=\sum_{k=m-r}^{n-r}\left(\begin{array}{c}n \\ k\end{array}\right) S_{n-k}^{(r)} S_{k}^{(m-r)}$ the result of the summation is

$$
a_{m}=\frac{(-1)^{m}}{m !} \prod_{j=0}^{m-2}(m p-j)
$$

Solving now for $\hat{v}=\partial_{\hat{u}} W$ and integrating we get, up to trivial integration constants, the superpotential

$$
W=\sum_{m=1}^{\infty} \frac{1}{m m !} \prod_{j=1}^{m-1}(m p-j) e^{m u}
$$

We can write this in the general form (2.11). In the case at hand that is given by 
$W=\sum_{m, k} \frac{N_{m}}{k^{2}} e^{k m \hat{u}}$ which yields the following integers for $N_{m}$ :

$$
\begin{aligned}
N_{1} & =(-1)^{p} \\
N_{2} & =-\frac{p}{2}+\frac{1-(-1)^{p}}{4}=-\left[\frac{p}{2}\right] \\
N_{3} & =\frac{(-1)^{p}}{2} p(p-1) \\
N_{4} & =-\frac{1}{3}(2 p-1)(p-1) p \\
N_{5} & =\frac{5}{24}(-1)^{p} p(p-1)\left(5 p^{2}-5 p+2\right) \\
N_{6} & =\frac{1}{20}(p-1) p\left(36 p^{3}-54 p^{2}+31 p-9\right) \\
N_{7} & =\frac{7}{720}(-1)^{p} p(p-1)\left(343 p^{4}-686 p^{3}+539 p^{2}-196 p+36\right) \\
\quad &
\end{aligned}
$$

To show integrality for a given expression $N_{m}(p)$ for all $p \in \mathbb{Z}$, one may factorize the denominator into $\prod_{i} p_{i}^{n_{i}}$ with $p_{i}$ prime and check that one can factor $p_{i}^{n_{i}}$ from the numerator for all $p=p_{i}^{n_{i}} n-k_{i}$ with $k_{i}=1, \ldots, p_{i}^{n_{i}}$. That has been checked for $m \leq 50$.

Note that $N_{m}$ is a polynomial in $p$ of degree $m-1$. As discussed in section 5 , the $p$ dependence of the superpotential for this particular Lagrangian brane (viewed as a large $t$ limit of the $O(-1) \oplus O(-1) \rightarrow \mathbf{P}^{1}$ geometry) can be mapped to the framing ambiguity of the unknot for the Chern-Simons theory. In appendix A we discuss this computation and verify that the large $N$ Chern-Simons computation leads to a polynomial of degree $m-1$ in $p$ for $N_{m}$. Moreover we have verified that for $N_{i}$ for $i=1,2,3$ the $p$ dependence of the framing choice agrees with the above result. We also have shown from the computation of the framing dependence of the CS theory that for all $m$ the coefficient of the leading term $p^{m-1}$ is given by $m^{m-2} / m$ ! in agreement with the above result.

Results of the prediction of [4] for the topological string amplitudes for the case of the unknot have recently been verified mathematically using localization methods in [32] [33]. In verifying the predictions of [4] there were some choices made for the toric action in these works. The authors of [32] have recently checked and verified that the integer choice of ambiguity changes the topological string amplitudes exactly 
as predicted by the above results $\mathrm{\theta}$. Moreover this ambiguity arises from the boundary of moduli space of Riemann surfaces with holes, as we already discussed.

\section{2. $\mathcal{O}(K) \rightarrow \mathbf{P}^{\mathbf{1}} \times \mathbf{P}^{\mathbf{1}}$}

This manifold involves a compact $\mathbf{P}^{1} \times \mathbf{P}^{1}$ geometry inside a CY 3-fold with a non-compact direction given by the canonical line bundle. It can be described by a linear sigma model with $G=U(1)^{2}$ and five chiral fields $X^{i}$, for $i=0, \ldots 4$ with charges $Q^{1}=(-2,1,1,0,0)$ and $Q^{2}=(-2,0,0,1,1)$ which leads to the $D$-terms

$$
\left|X^{1}\right|^{2}+\left|X^{2}\right|^{2}-2\left|X^{0}\right|^{2}=r_{t} \quad\left|X^{3}\right|^{2}+\left|X^{4}\right|^{2}-2\left|X^{0}\right|^{2}=r_{s}
$$

The solutions to the D-term equation, projected to the toric base are given in Fig.13. The $\mathbf{F}_{\mathbf{0}}=\mathbf{P}^{\mathbf{1}} \times \mathbf{P}^{\mathbf{1}}$ corresponds to the divisor class represented by $X^{0}=0$ and is visible in Fig.13 as the minimal parallelogram in the toric base. The fiber $O(K)$ corresponds to the normal direction. The special Lagrangian D-branes of the Amodel for this Calabi-Yau have inequivalent phases, depending on where one puts the brane, together with a $\mathbf{Z}$-family of choices of framing in each. The D-brane charge can be taken to be $q_{1}=(-1,0,1,0,0), q_{2}=(-1,0,0,1,0)$ and so

$$
\left|X^{2}\right|^{2}-\left|X^{0}\right|^{2}=c^{1}, \quad\left|X^{3}\right|^{2}-\left|X^{0}\right|^{2}=c^{2}
$$

The constants $c_{i}$ are required to be chosen so that the Lagrangian D-brane lies on one dimensional toric edges, and different toric legs give rise to generally different phases of the theory.

The mirror variables $Y^{i}$ satisfy

$$
Y^{1}+Y^{2}-2 Y^{0}=-t, \quad Y^{3}+Y^{4}-2 Y^{0}=-s
$$

where the real parts of complex structure parameters $t, s$ measure the classical sizes of the two minimal $P^{1}$ 's in the toric base, $\operatorname{Re}(t)=r_{t}, \operatorname{Re}(s)=r_{s}$. The mirror manifold is given by

$$
x z=e^{u}+e^{-t-u}+e^{v}+e^{-s-v}+1
$$

5 We are grateful to S. Katz and C.-C. Liu for performing these computations upon our request. The form we have presented our answer (6.4) was chosen to simplify the comparison with their formula. 
where $Y^{2}=u, Y^{3}=v, Y^{0}=0$ and the two holomorphic constrains are solved in terms of these. The Riemann surface $\Sigma$ which is the configuration space of the mirror B-brane is given by $0=P(u, v)=e^{u}+e^{-t-u}+e^{v}+e^{-s-v}+1$. When viewed in terms of the single valued variables $e^{u}$ and $e^{v}, \Sigma$ is related to the $A$-model geometry by "thickening" of the one-dimensional edges of the toric diagram (or more precisely, their projection onto the $X^{0}=0$ plane). In the large radius limit $0 \ll t, s$ the A-model geometry becomes classical and the legs of the Riemann surface of the B-model become long and thin, so the A- and the B-model can be related already at the classical level.

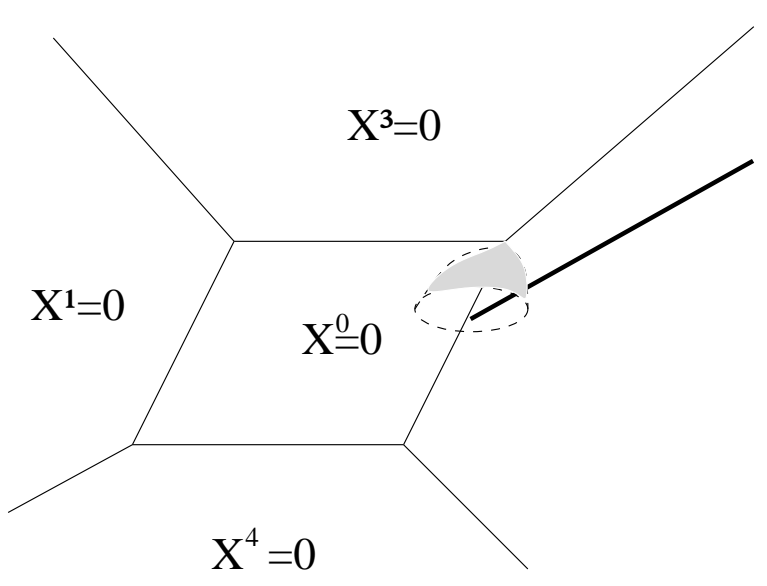

a.

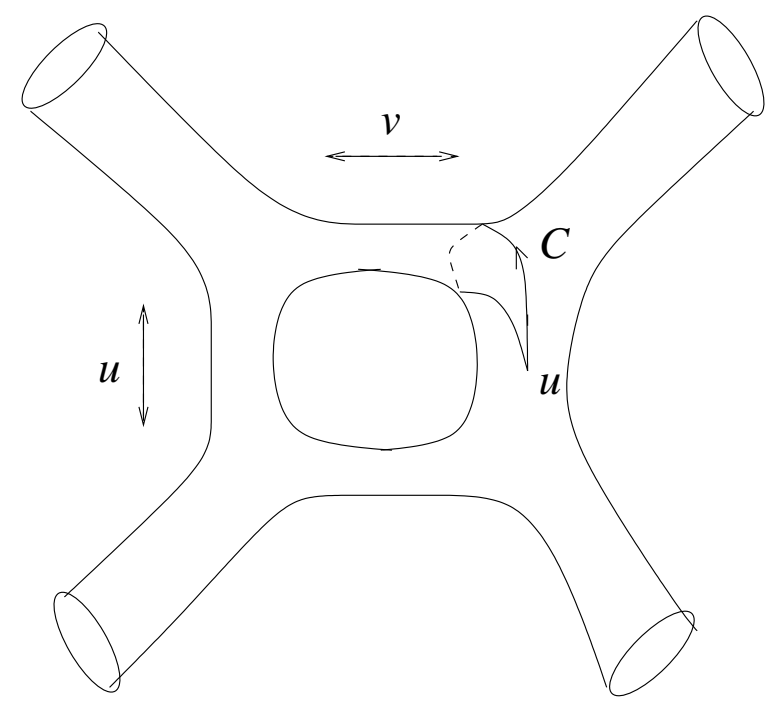

b.

Fig.13 The Figure a. depicts the base of the toric fibration for $X=O(K) \rightarrow \mathbf{P}^{\mathbf{1}} \times \mathbf{P}^{\mathbf{1}}$ together with a special Lagrangian brane in $X$. The minimal disk which ends on the A-brane in the figure wraps partly the $\mathbf{P}^{\mathbf{1}}$ over which the brane is. Figure b. depicts the Riemann surface $\Sigma$ of the mirror geometry, and the mirror B-brane as a choice of a point on $\Sigma$. D4 brane wrapping the disk in figure a. is mirror to a domain-wall which projects to path $\mathcal{C}$ in Figure $b$.

For example, the A-brane on Fig.13 is on the internal leg which is given by $c_{2}=0$, and $c_{1}$ in the $\left[0, r_{t}\right]$ interval. The large radius limit is, in addition to $r_{t}$ and $r_{s}$ being large, the limit of large disk size. For example in the regime $c_{1}<r_{t} / 2$ the basic disk is the one on the Fig.13a and the classical limit corresponds to $c_{1}$ being of order of $r_{t} / 2$. The size parameter $c_{1}$ together with the Wilson line $u=c_{1}+i \int A$ is 
the classical tension of the D4 brane domain wall wrapping this disk and ending on the D6 brane.

In the limit the A-model geometry is classical, it follows from the mirror map (2.3) that the mirror B-brane is located in the region on $\Sigma$ where $v$ is constant, $v \sim 0$, and $u$ a parameter which is large, $u \sim t / 2$. Away from the large radius limit, this deforms to a root of the equation $P(u, v)=0$. This has two solutions for $v$ at every value of $u$,

$$
v=v_{1,2}(u)=\log \left[\frac{1+e^{u}+e^{-t-u}}{2} \pm \frac{\sqrt{\left(1+e^{u}+e^{-t-u}\right)^{2}-4 e^{-s}}}{2}\right]+i \pi
$$

and the mirror B-brane propagates along a region of the root $v=v_{1}(u)$. As we discussed above, the superpotential on this leg is given by

$$
W(u)=\int v_{1}(u) d u
$$

This vanishes in the classical limit since $\partial_{u} W=v_{1}(u) \rightarrow 0$.

The flat coordinate computes the tension $\hat{u}$ of the D4 brane domain wall wrapped on the basic disk as in Fig.13a, and we will compute this by computing the tension of the mirror B-brane domain wall. As explained in section 4. the mirror domain wall projects to the loop $\mathcal{C}$ on the Riemann surface which starts at the location of the B-brane at a fixed $u$, winds around $v \rightarrow v+2 \pi i$ before "attaching" again (see Fig.13b). In this configuration, the tension is given by a classical integral on the Riemann surface

$$
\hat{u}=\frac{1}{2 \pi i} \int_{\mathcal{C}} v_{1}(u) d u
$$

The period integral $\frac{1}{2 \pi i} \int_{\mathcal{C}} v_{1}(u) d u$ is, as explained in Section 4 , is the sum of two pieces. There is the classical contribution to the domain wall tension which is equal to $\frac{1}{2 \pi i} \Delta\left(v_{1} u\right)=u$ as the the initial and final end-point of $\mathcal{C}$ differ by $v \rightarrow v+2 \pi i$. The quantum correction to the BPS mass comes from the "small period", the contour integral around $\beta_{1}$ cycle (see Fig.14) of $\frac{1}{2 \pi i} \int_{\beta_{1}} u d v$. 

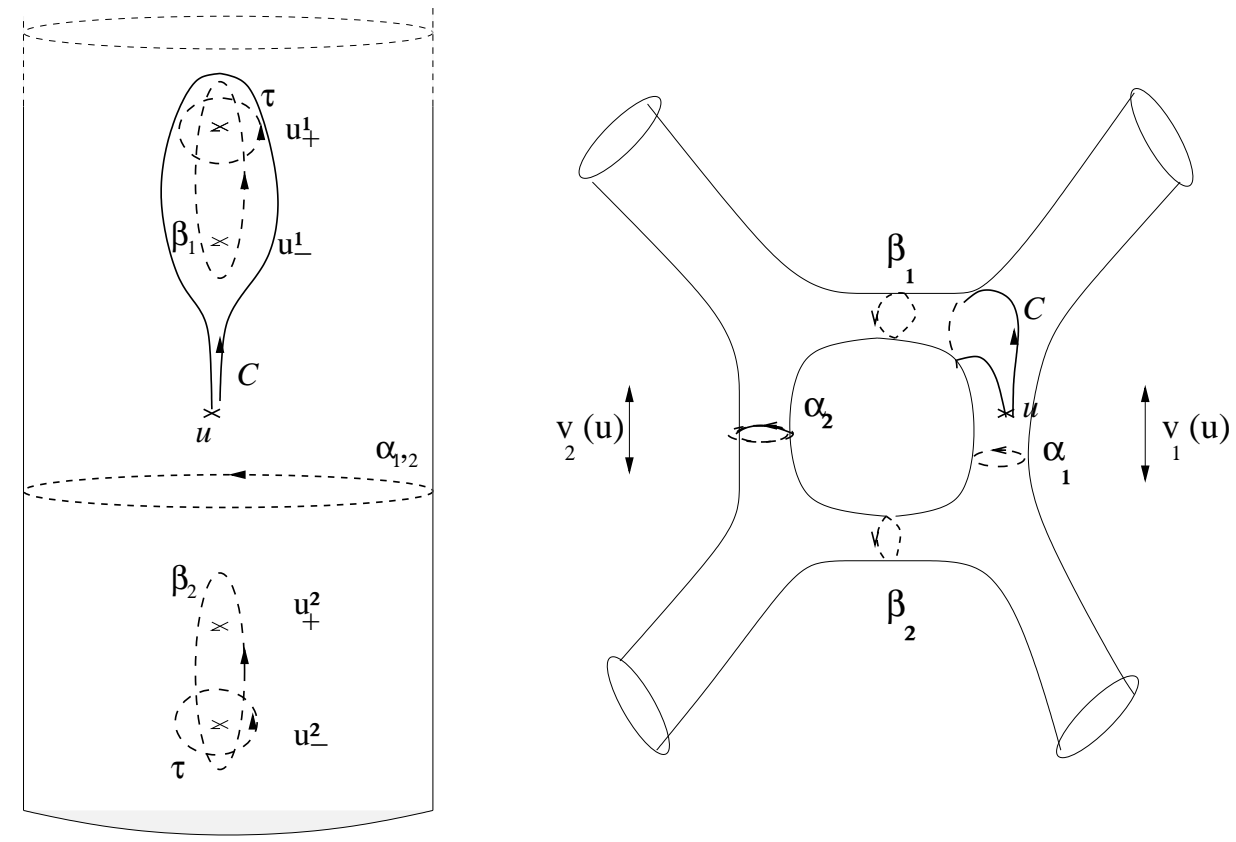

Fig.14 Riemann surface $\Sigma: P(u, v)=0$ can be viewed as a branched cover of the $u$-cylinder by two solutions $v_{1,2}(u)$. The solutions are branched over 4 points $u_{1,2}^{ \pm}$, with monodromies $\tau_{1,2}$ which exchanges $v_{1} \leftrightarrow v_{2}$, and $\beta_{1,2}$ which take $v_{1,2} \rightarrow v_{1,2}+2 \pi i$ corresponding to the $v$-cylinders which glue the two roots on the figure to the right. Monodromies $\alpha_{1,2}$ arise from periodicity of $u$ itself.

The small periods can be found as follows. Notice that since $v_{1}+v_{2}=-s+2 \pi i$, the sum of two periods around $u \rightarrow u+2 \pi i$ is

$$
\frac{1}{2 \pi i} \int_{\alpha_{1}} v_{1} d u+\frac{1}{2 \pi i} \int_{\alpha_{2}} v_{2} d u=s+2 \pi i .
$$

On the other hand, the closed string period $\hat{s}$, that measures the mass of the D4 brane wrapping a $\mathbf{P}^{\mathbf{1}}$ of size $r_{s}$ can also be expressed in terms of small periods as it is computed along the contour $\alpha_{s}=\alpha_{1}-\alpha_{2}$, (orientations are fixed up to an over-all sign by the requiring that both $u$ and $v$ have trivial monodromy around $\beta_{s}$ ),

$$
\frac{1}{2 \pi i} \int_{\alpha_{1}} v_{1} d u-\frac{1}{2 \pi i} \int_{\alpha_{2}} v_{2} d u=-\hat{s} .
$$

From this we can find for example the small period along $\alpha_{1}$ as $\frac{s-\hat{s}}{2}+i \pi=$ $\frac{1}{2 \pi i} \int_{\alpha_{1}} v_{1} d u$.

6 This method of calculating the domain-wall tension gives the result up to factors of $i \pi$. The direct evaluation of the period around $\mathcal{C}$ can be done, and it determines the answer to be the one we presented above. 


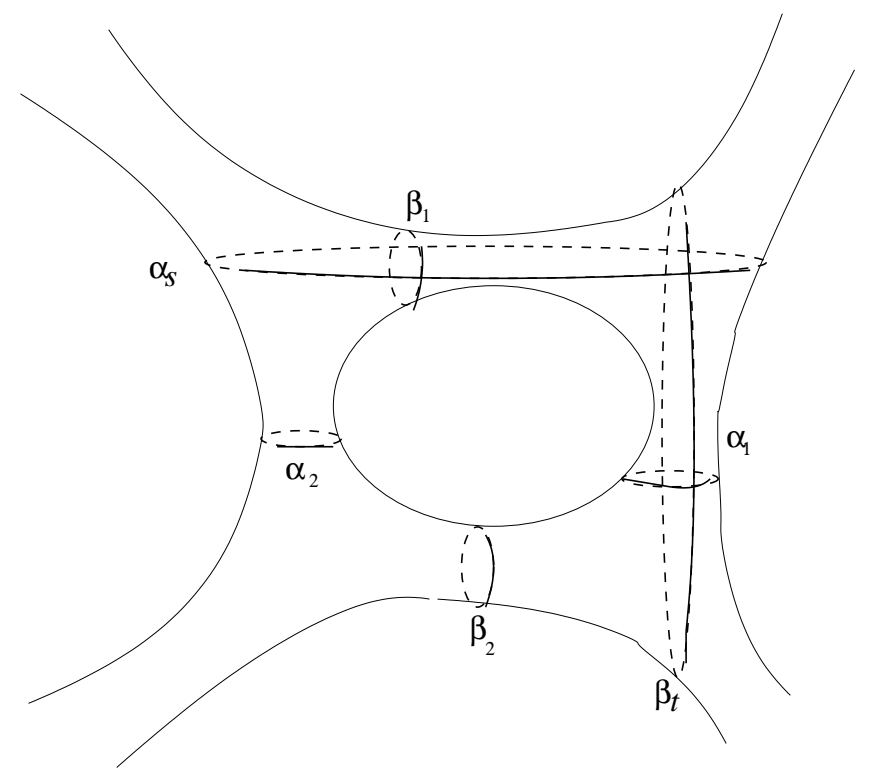

Fig.15 Closed string periods $\beta_{t}, \beta_{s}$ are linear combinations of small periods $\alpha_{i}, \beta_{i}$ for $i=1,2$ which come from periodicity of $u$ and $v$ variables.

For the $B$-brane at hand we need the small period around $\beta_{1}$ cycle on the $v$-leg, and by computation analogous to the one we just did,

$$
\hat{u}=u+\frac{t-\hat{t}}{2}+\pi i \text {. }
$$

In fact the small period of $\alpha_{1}$ is the correction for the B-brane on the other leg of the Riemann surface - the leg parameterized by $v$ which corresponds to the phase $I I$ (see Fig.16). In this phase we have therefore

$$
\hat{v}=v+\frac{s-\hat{s}}{2}+i \pi .
$$

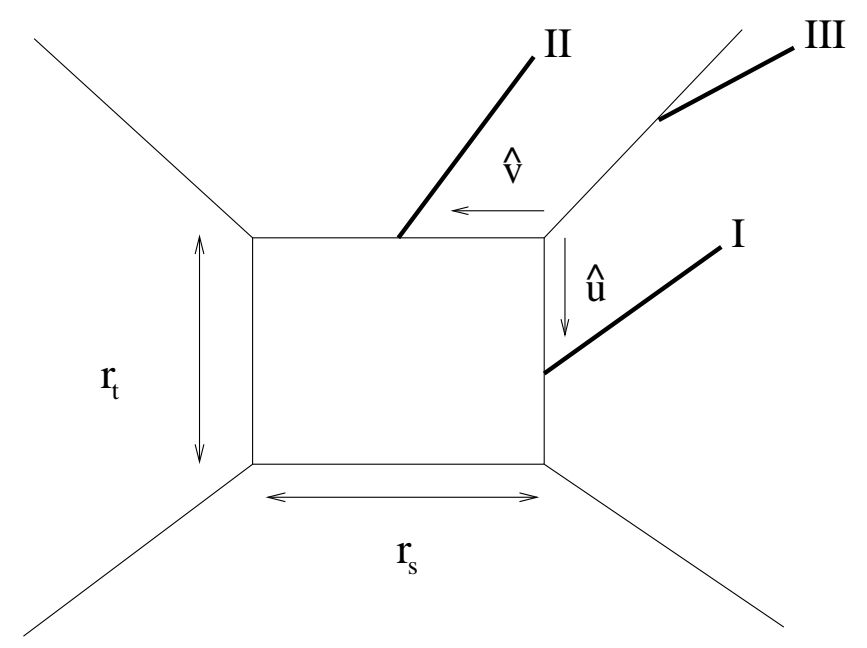


Fig.16 Three phases of the $A$-brane on $O(K) \rightarrow \mathbf{P}^{\mathbf{1}} \times \mathbf{P}^{\mathbf{1}}$. Phases $I, I I$ are related by the exchange of the two $\mathbf{P}^{\mathbf{1}}$ 's in the base, and this is reflected in the disc domain wall numbers.

According to the discussion in the $\mathbf{C}^{\mathbf{3}}$ case and which carries over here as well, the correction terms we computed

$$
\Delta_{u}=\frac{t-\hat{t}}{2}+i \pi \quad \Delta_{v}=\frac{s-\hat{s}}{2}+i \pi
$$

form a doublet under the $\mathrm{SL}(2, \mathbb{Z})$ transformations, just as $u, v$ do. Consider now an $\mathrm{SL}(2, \mathbb{Z})$ group element which takes:

$$
u \rightarrow u^{\prime}=u-v, \quad v \rightarrow v^{\prime}=v
$$

The new equation of the curve becomes

$$
e^{u^{\prime}-v^{\prime}}+e^{-t-u^{\prime}+v^{\prime}}+e^{v^{\prime}}+e^{-s-v^{\prime}}+1=0
$$

This change of coordinates does not change the classical value of the superpotential in phase $I$ (the phase originally parameterized by $u$ ) since $v^{\prime}=v \sim 0$ but corresponds to a different choice of framing with $n=-1$ which changes the fluctuating field on the brane from $u$ to $u^{\prime}=u-v$. The quantum corrected domain wall tension is now

$$
\Delta_{u^{\prime}}=\Delta_{u}-\Delta_{v}
$$

For the brane in phase $I I$ it is no longer true that the classical superpotential is zero, since $u^{\prime}=u-v$ is not zero after the $\operatorname{SL}(2, \mathbb{Z})$ transformation.

With this choice of parameterization, consider the superpotential

$$
W=\int u^{\prime}\left(v^{\prime}\right) d v^{\prime}
$$

with $u^{\prime}(v)$ obtained by solving $P\left(u^{\prime}, v\right)=0$. This superpotential has no classical piece, as $v^{\prime} \rightarrow \infty, u^{\prime} \sim 0$ is on the curve. In fact, $u^{\prime} \sim 0$ is the equation of the outer leg of $\Sigma$ (see Fig. 16), so this computes the superpotential of the brane in phase III. The domain wall tension in the phase III (the outer leg) is given by

$$
\Delta_{v^{\prime}}=\Delta_{v}=\frac{s-\hat{s}}{2}+i \pi
$$

7 As we will see when we discuss the closed string flat coordinates in more detail below, $\Delta_{u^{\prime}}$ turns out to be zero. 
To compute disk numbers in these various phases, we need to write the superpotential in each case in terms of the open and closed string flat coordinates.

The closed string flat coordinates have a complicated dependence on the classical, linear-sigma model, coordinates $t$. Quite analogously to what we found in the open string, the $A$-model closed string amplitudes have integrality properties when expanded in terms of flat coordinates which measure the BPS mass of D2-branes wrapped on rational curves in $X$. The corrected quantities are related transcendentally by the mirror map $\hat{t_{i}}=\hat{t_{i}}\left(t_{j}\right)$ to the complex structure variable $z_{i}=e^{-t_{i}}$. In fact, as discussed above, $\hat{t}$ and $\hat{s}$ are among the periods of the Riemann surface $\Sigma$. Alternatively, they can be obtained from the solutions to Picard-Fuch's equations $\mathcal{L}_{i} f=0$,

$$
\mathcal{L}_{i}=\prod_{Q_{i}>0}\left(\partial_{i}\right)^{Q_{i}}-\prod_{Q_{i}<0}\left(\partial_{i}\right)^{-Q_{i}}
$$

where $\partial_{i}=\partial / \partial z^{i}$. For the $O(K) \rightarrow P^{1} \times P^{1}$ the linear differential operators $\mathcal{L}_{t, s}$ are:

$$
\begin{aligned}
& \mathcal{L}_{t}=\theta_{t}^{2}-2 z_{t}\left(\theta_{t}+\theta_{s}\right)\left(2 \theta_{s}+2 \theta_{t}+1\right) \\
& \mathcal{L}_{s}=\theta_{s}{ }^{2}-2 z_{s}\left(\theta_{t}+\theta_{s}\right)\left(2 \theta_{s}+2 \theta_{t}+1\right),
\end{aligned}
$$

where $\theta_{t, s}=z_{t, s} \partial / \partial z_{t, s}$. There is a constant solution $f_{0}=1$ and near $z_{t}=z_{s}=0$ there are two logarithmic solutions $f_{1}, f_{2}$. The flat coordinates $\hat{t}, \hat{s}$ are given by (linear combinations) of ratios of the periods $f_{i} / f_{0}, i=1,2$ picked by correct classical behavior:

$$
\begin{aligned}
& \hat{t}=t-\left(2 z_{t}+2 z_{s}+3 z_{t}^{2}+12 z_{t} z_{s}+3 z_{s}^{2}+O\left(z^{3}\right)\right) \\
& \hat{s}=s-\left(2 z_{t}+2 z_{s}+3 z_{t}^{2}+12 z_{t} z_{s}+3 z_{s}^{2}+O\left(z^{3}\right)\right) .
\end{aligned}
$$

Note that the solutions are symmetric in $z_{t, s}$ except for the logarithmic term. To expand the disk amplitude in terms of the flat coordinates we need the inverse relations $z_{t, s}\left(q_{t, s}\right)$ for $q_{t}=e^{-\hat{t}}$ and $q_{s}=e^{-\hat{s}}$. The first few terms of the expansion are

$$
\begin{aligned}
& z_{s}=q_{s}-2 q_{s}^{2}-2 q_{s} q_{2}+3 q_{s}^{3}+3 q_{s} q_{t}^{2}-4 q_{s}^{4}-4 q_{s}^{3} q_{t}-4 q_{s}^{2} q_{t}^{2}-4 q_{s} q_{t}^{3}+O\left(q^{5}\right) \\
& z_{t}=q_{t}-2 q_{s} q_{t}-2 q_{t}^{2}+3 q_{s}^{2} q_{t}+3 q_{t}^{3}-4 q_{s}^{3} q_{t}-4 q_{s}^{2} q_{t}^{2}-4 q_{s} q_{t}^{3}-4 q_{t}^{4}+O\left(q^{5}\right) .
\end{aligned}
$$

where $q_{t}=e^{-\hat{t}}$ and $q_{s}=e^{-\hat{s}}$.

To get the disk numbers the only remaining task is to expand the $W(u)$ in terms of open and closed string flat coordinates. The integrality of the disk amplitude (2.11) implies that we write $\partial_{\hat{u}} W(\hat{u})=\hat{v}$ as

$$
\partial_{\hat{u}} W(\hat{u})=-\sum_{\substack{k_{s}=0, k_{t}=0 \\ m=-k_{t}}}^{\infty} m N_{k_{s}, k_{t}, m} \log \left(1-q_{s}^{k_{s}} q_{t}^{k_{t}} e^{m \hat{u}}\right)
$$


In phase $I$ we get the following values for numbers of primitive disks $N_{k_{s}, k_{t}, m}$ :

\begin{tabular}{|c|rrrrrr|}
\hline$m=1$ & \multicolumn{7}{|c|}{} \\
\hline$k_{s}$ & $k_{t}=0$ & 1 & 2 & 3 & 4 & 5 \\
\hline 0 & 1 & 0 & 0 & 0 & 0 & 0 \\
1 & 1 & 2 & 3 & 4 & 5 & 6 \\
2 & 1 & 10 & 45 & 140 & 350 & 756 \\
3 & 1 & 30 & 300 & 1776 & 7650 & 26532 \\
4 & 1 & 70 & 1332 & 13400 & 91070 & 472368 \\
5 & 1 & 140 & 4590 & 72856 & 736270 & 5437530 \\
\hline
\end{tabular}

\begin{tabular}{|c|c|c|c|c|c|c|}
\hline$m=2$ & & & & & & \\
\hline$k_{s}$ & $k_{t}=0$ & 1 & 2 & 3 & 4 & 5 \\
\hline 0 & 0 & 0 & 0 & 0 & 0 & 0 \\
\hline 1 & 1 & 2 & 3 & 4 & 5 & 6 \\
\hline 2 & 2 & 16 & 62 & 180 & 428 & 896 \\
\hline 3 & 4 & 70 & 552 & 2856 & 11280 & 36828 \\
\hline 4 & 6 & 224 & 3130 & 26336 & 159078 & 759200 \\
\hline 5 & 9 & 588 & 13420 & 171720 & 1503135 & 10016490 \\
\hline
\end{tabular}

\begin{tabular}{|c|rrrrrr|}
\hline$m=3$ & \multicolumn{7}{|c|}{$k^{\prime}$} & & & & \\
\hline$k_{s}$ & $k_{t}=0$ & 1 & 2 & 3 & 4 & 5 \\
\hline 0 & 0 & 0 & 0 & 0 & 0 & 0 \\
1 & 1 & 2 & 3 & 4 & 5 & 6 \\
2 & 4 & 24 & 85 & 230 & 525 & 1064 \\
3 & 11 & 146 & 977 & 4542 & 16644 & 51420 \\
4 & 25 & 618 & 6975 & 50912 & 278134 & 1231230 \\
5 & 49 & 2070 & 36637 & 395818 & 3068331 & 18655290 \\
\hline
\end{tabular}

\begin{tabular}{|c|rrrrrr|}
\hline$m=4$ & \multicolumn{7}{|c|}{} & \multicolumn{1}{|c|}{$\mid$} & 5 \\
\hline$k_{s}$ & $k_{t}=0$ & 1 & 2 & 3 & 4 & 0 \\
\hline 0 & 0 & 0 & 0 & 0 & 0 & 6 \\
1 & 1 & 2 & 3 & 4 & 5 & 1260 \\
2 & 6 & 34 & 112 & 290 & 638 & 71400 \\
3 & 25 & 276 & 1645 & 7040 & 24246 & 1979098 \\
4 & 76 & 1498 & 14496 & 94830 & 476900 & \\
5 & 196 & 6248 & 91935 & 870220 & 6103867 & 34309080 \\
\hline
\end{tabular}




\begin{tabular}{|c|rrrrrr|}
\hline$m=5$ & \multicolumn{7}{|c|}{} \\
\hline$k_{s}$ & $k_{t}=0$ & 1 & 2 & 3 & 4 & 5 \\
\hline 0 & 0 & 0 & 0 & 0 & 0 & 0 \\
1 & 1 & 2 & 3 & 4 & 5 & 6 \\
2 & 9 & 46 & 145 & 360 & 770 & 1484 \\
3 & 49 & 482 & 2640 & 10592 & 34674 & 98028 \\
4 & 196 & 3270 & 28240 & 169402 & 795998 & 3126928 \\
5 & 635 & 16642 & 213083 & 1816038 & 11729677 & 61675880 \\
\hline
\end{tabular}

\begin{tabular}{|c|rrrrrr|}
\hline$m=6$ & \multicolumn{7}{|c|}{3} & 5 \\
\hline$k_{s}$ & $k_{t}=0$ & 1 & 2 & 3 & 4 & 0 \\
\hline 0 & 0 & 0 & 0 & 0 & 0 & 6 \\
1 & 1 & 2 & 3 & 4 & 5 & 1736 \\
2 & 12 & 60 & 182 & 440 & 918 & 132732 \\
3 & 87 & 790 & 4060 & 15478 & 48600 & 4840248 \\
4 & 440 & 6560 & 51906 & 290600 & 1290870 & 107979508 \\
5 & 1764 & 40050 & 460355 & 3604656 & 21737688 & 102 \\
\hline
\end{tabular}

Tab.1 The disk domain wall degeneracies for brane $I$ in the $O(K) \rightarrow \mathbf{P}^{\mathbf{1}} \times \mathbf{P}^{\mathbf{1}}$ geometry for $m>0$. Exchanging $s$ with $t$ yields the result for brane $I I$.

There is a symmetry which relates the numbers of disk instantons with negative $m$ to those above. We have

$$
N_{k_{s}, k_{t},-m}= \begin{cases}0 & \text { if } k_{t}-m<0 \\ -N_{k_{s}, k_{t}-m, m} & \text { if } k_{t}-m \geq 0\end{cases}
$$

so for e.g

\begin{tabular}{|c|rrrrrr|}
\hline$m=-1$ & \multicolumn{7}{|c|}{} \\
\hline$k_{s}$ & $k_{t}=0$ & 1 & 2 & 3 & 4 & 5 \\
\hline 0 & 0 & -1 & 0 & 0 & 0 & 0 \\
1 & 0 & -1 & -2 & -3 & -4 & -5 \\
2 & 0 & -1 & -10 & -45 & -140 & -350 \\
3 & 0 & -1 & -30 & -300 & -1776 & -7650 \\
4 & 0 & -1 & -70 & -1332 & -13400 & -91070 \\
5 & 0 & -1 & -140 & -4590 & -72856 & 736270 \\
\hline
\end{tabular}

Tab.2 Disk degeneracies for brane $I$ in the $O(K) \rightarrow \mathbf{P}^{\mathbf{1}} \times \mathbf{P}^{\mathbf{1}}$ geometry for $m<0$.

The numbers of primitive disks for the brane III are 


\begin{tabular}{|c|rrrrrr|}
\hline$m=1$ & \multicolumn{1}{|c|}{} & & & & \\
\hline$k_{s}$ & $k_{t}=0$ & 1 & 2 & 3 & 4 & 5 \\
\hline 0 & 2 & 2 & 2 & 2 & 2 & 2 \\
1 & 0 & 2 & 12 & 40 & 100 & 210 \\
2 & 0 & 2 & 40 & 310 & 1520 & 5628 \\
3 & 0 & 2 & 100 & 1520 & 12908 & 76488 \\
4 & 0 & 2 & 210 & 5628 & 91070 & 680940 \\
5 & 0 & 2 & 392 & 17184 & 353316 & 4515558 \\
\hline
\end{tabular}

\begin{tabular}{|c|rrrrrr|}
\hline$m=2$ & & & & & \\
\hline$k_{s}$ & $k_{t}=0$ & 1 & 2 & 3 & 4 & 5 \\
\hline 0 & 0 & 0 & -2 & -4 & -8 & -12 \\
1 & 0 & 0 & -4 & -32 & -140 & -448 \\
2 & 0 & 0 & -8 & -140 & -1188 & -6580 \\
3 & 0 & 0 & -12 & -448 & -6580 & -58240 \\
4 & 0 & 0 & -28 & -1176 & -27840 & -370428 \\
5 & 0 & 0 & -24 & -2688 & -97020 & -1859648 \\
\hline
\end{tabular}

\begin{tabular}{|c|c|c|c|c|c|c|}
\hline$m=3$ & & & & & & \\
\hline$k_{s}$ & $k_{t}=0$ & 1 & 2 & 3 & 4 & 5 \\
\hline 0 & 0 & 0 & 0 & 2 & 10 & 28 \\
\hline 1 & 0 & 0 & 0 & 10 & 100 & 540 \\
\hline 2 & 0 & 0 & 0 & 28 & 540 & 5012 \\
\hline 3 & 0 & 0 & 0 & 62 & 2100 & 317072 \\
\hline 4 & 0 & 0 & 0 & 120 & 6600 & 147420 \\
\hline 5 & 0 & 0 & 0 & 210 & 17820 & 576212 \\
\hline
\end{tabular}

\begin{tabular}{|c|rrrrrr|}
\hline$m=4$ & & & & & & \\
\hline$k_{s}$ & $k_{t}=0$ & $\ldots$ & 4 & 5 & 6 & 7 \\
\hline 0 & 0 & $\ldots$ & 0 & -4 & -28 & -104 \\
1 & 0 & $\ldots$ & 0 & -28 & -336 & -2156 \\
2 & 0 & $\ldots$ & 0 & -104 & -2156 & -21888 \\
3 & 0 & $\ldots$ & 0 & -300 & -9856 & -149940 \\
4 & 0 & $\ldots$ & 0 & -720 & -36036 & -787640 \\
5 & 0 & $\ldots$ & 0 & -1540 & -112112 & -3406480 \\
\hline
\end{tabular}




\begin{tabular}{|c|rrrrrr|}
\hline$m=5$ & & & & & \\
\hline$k_{s}$ & $k_{t}=0$ & $\ldots$ & 5 & 6 & 7 & 8 \\
\hline 0 & 0 & $\ldots$ & 0 & 10 & 84 & 396 \\
1 & 0 & $\ldots$ & 0 & 84 & 1176 & 8736 \\
2 & 0 & $\ldots$ & 0 & 396 & 8736 & 96660 \\
3 & 0 & $\ldots$ & 0 & 1386 & 45864 & 724800 \\
4 & 0 & $\ldots$ & 0 & 4004 & 191100 & 4273840 \\
5 & 0 & $\ldots$ & 0 & 10090 & 672672 & - \\
\hline
\end{tabular}

\begin{tabular}{|c|rrrrrr|}
\hline$m=6$ & & & & & \\
\hline$k_{s}$ & $k_{t}=0$ & - & 6 & 7 & 8 & 9 \\
\hline 0 & 0 & $\ldots$ & 0 & -26 & -264 & -1504 \\
1 & 0 & $\ldots$ & 0 & -264 & -4224 & -35640 \\
2 & 0 & $\ldots$ & 0 & -1504 & -35640 & -427540 \\
3 & 0 & $\ldots$ & 0 & -6228 & -211200 & -3484800 \\
4 & 0 & $\ldots$ & 0 & -21028 & -987360 & - \\
5 & 0 & $\ldots$ & 0 & -61152 & - & - \\
\hline
\end{tabular}

Tab.3 The disk degeneracies for brane III in the $O(K) \rightarrow \mathbf{P}^{\mathbf{1}} \times \mathbf{P}^{\mathbf{1}}$ geometry for $m>0$.

Up to the invariant $N_{0,0,1}=2$ we have $N_{k_{s}, k_{t}, m}=0$ for $k_{t}<m$ and for $k_{t} \geq m$ there is a symmetry

$$
N_{k_{s}, k_{t}, m}=N_{k_{t}-m, k_{s}+m, m}
$$

which reflects the exchange symmetry of $s$ and $t$ for the brane in phase III. The instantons with negative $m$ are absent for $k_{s} \leq|m|$ and their numbers are related to those with positive $m$ by

$$
N_{k_{s}, k_{t},-m}=-N_{k_{s}+m, k_{t}-m, m},
$$

e.g.

\begin{tabular}{|c|rrrrrr|}
\hline$m=-1$ & \multicolumn{7}{|c|}{} \\
\hline$k_{s}$ & $k_{t}=0$ & 1 & 2 & 3 & 4 & 5 \\
\hline 0 & 0 & 0 & 0 & 0 & 0 & 0 \\
1 & -2 & -2 & -2 & -2 & -2 & -2 \\
2 & -2 & -12 & -40 & -100 & -210 & -392 \\
3 & -2 & -40 & -310 & -1520 & -5628 & -17184 \\
4 & -2 & -100 & -1520 & -12908 & -76488 & -353316 \\
5 & -2 & -210 & -5628 & -76488 & -680940 & -4515558 \\
\hline
\end{tabular}


Tab.4 The disk degeneracies for brane III in the $O(K) \rightarrow \mathbf{P}^{\mathbf{1}} \times \mathbf{P}^{\mathbf{1}}$ geometry for $m<0$.

6.3. $\mathcal{O}(-3) \rightarrow \mathbf{P}^{2}$

The linear sigma model for this geometry has $G=U(1)$ and four matter fields with charges $Q=(-3,1,1,1)$

$$
\left|X^{1}\right|^{2}+\left|X^{2}\right|^{2}+\left|X^{3}\right|^{2}-3\left|X^{0}\right|^{2}=r
$$

The base of the toric fibration is shown in the Fig.17.

The D-brane charge can be taken to be $q_{1}=(1,0,-1,0), q_{2}=(0,1,-0,-1)$ (the choice is unique in all local models) and so

$$
\left|X^{1}\right|^{2}-\left|X^{0}\right|^{2}=c^{1}, \quad\left|X^{2}\right|^{2}-\left|X^{0}\right|^{2}=c^{2}
$$

Consider three phases of the A-brane that are visible classically (see Fig. 17).

$$
\begin{aligned}
\text { Phase I : } & r_{t}>c^{1}>0, \quad c^{2}=0 \\
\text { Phase II : } & c^{1}=0, \quad r_{t}>c^{2}>0 \\
\text { Phase III : } & c^{1}=c^{2}, \quad 0<c^{1}
\end{aligned}
$$

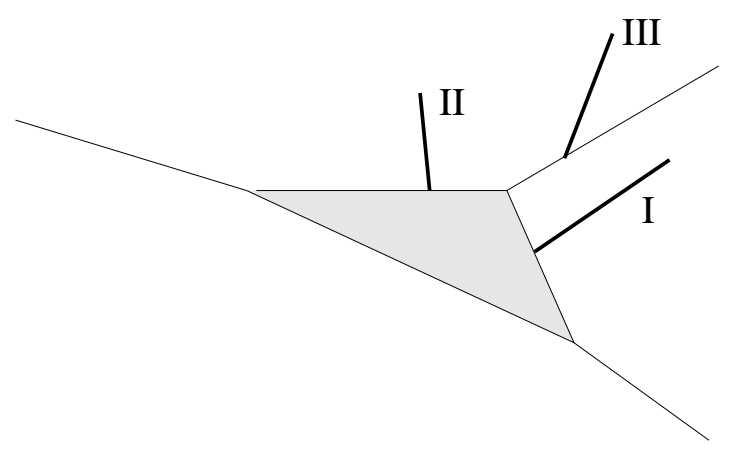

Fig. 17 Three phases of the $A$-brane on $O(-3) \rightarrow \mathbf{P}^{\mathbf{2}}$. Phases $I$ and $I I$ are related by $\mathbf{Z}_{\mathbf{3}}$ symmetry of the $\mathbf{P}^{2}$.

As the branes in phases $I$ and $I I$ are related by the $\mathbf{Z}_{3}$ symmetry of $\mathbf{P}^{2}$, the special Lagrangian D-branes of the A-model for this Calabi-Yau have two inequivalent phases, together with a $\mathbf{Z}$-family of choices of framing in each.

The mirror $B$-model geometry (see e.g. [34] [35] [36]) can be written as

$$
x z=e^{u}+e^{v}+e^{-t-u-v}+1
$$


where we have "solved" $Y^{1}+Y^{2}+Y^{3}=-t+3 Y^{0}$ by $Y^{0}=0, Y^{1}=u, Y^{2}=v$ and $Y_{3}=-t-u-v$.

In terms of these coordinates, the brane in phase $I$ propagates on the internal leg of the Riemann surface where $v \sim 0$ and $u$ large of order $-t / 2$, the brane in phase $I I$ is on $u \sim 0$, and $v \sim t / 2$, and the brane on external leg has $u \sim v$, and both are large.

In the variables of (6.14) the classical superpotential vanishes in phase $I$ and $W(u)=\int v(u) d u$ computes the disk instanton generated superpotential.

To compute disk numbers we need the flat coordinate. According to discussion in section 5, this is given by the difference of superpotentials on the two sides of the domain wall as computed along the contour $C$ on Fig.18. The non-trivial contribution to $\hat{u}$ is the exponentially suppressed shift which comes from the small period on the Fig.18.

$$
\hat{u}=\int_{C} v(u) d u=u+\Delta_{u}
$$

We find, using the same kind of methods discussed for the $\mathbf{P}^{1} \times \mathbf{P}^{1}$ example that,

$$
\Delta_{u}=\frac{t-\hat{t}}{3}+i \pi
$$

where $\hat{t}$ is the closed string period on contour $\alpha_{1}$ in Fig.18.

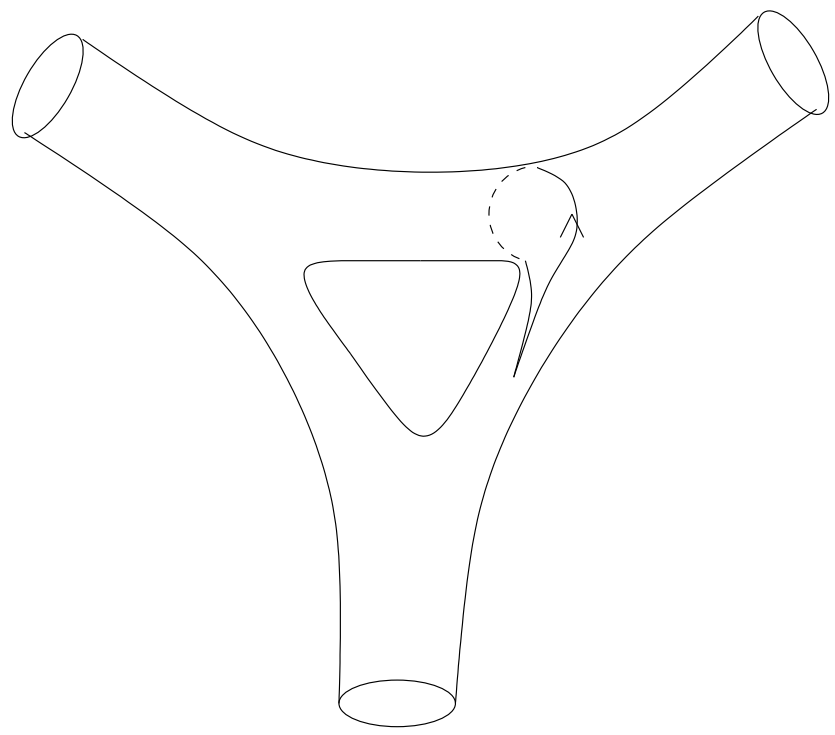

Fig.18 The curve $P(u, v)=0$ associated to the mirror of $O(-3) \rightarrow \mathbf{P}^{\mathbf{2}}$. 
The closed string period can be computed either directly by integration on $\Sigma$, or from the Picard-Fuchs equation with $\mathcal{L}=\theta^{3}+3 z \theta(3 \theta+1)(3 \theta+2)$ where $\theta=z \partial_{z}$, and $z=e^{-t}$. The solutions to the Picard-Fuchs equation can be expressed e.g. as Mejer G-functions [37 and

$$
\hat{t}=t-\sum_{n=1}^{\infty} \frac{(-1)^{n}}{n} \frac{(3 n) !}{(n !)^{3}} z^{n}
$$

The inverse $z(\hat{t})$ relation is:

$$
z=q+6 q^{2}+9 q^{3}+56 q^{4}-300 q^{5}+3942 q^{6}+\ldots
$$

where $q=e^{-\hat{t}}$.

After rewriting $W(u, t)$ in terms of the flat coordinates $\hat{u}, \hat{t}$, and expanding as in (2.11) we obtain the following integer invariants

\begin{tabular}{|c|rrrrrrrrr|}
\hline$m$ & $k=0$ & 1 & 2 & 3 & 4 & 5 & 6 & 7 & 8 \\
\hline-5 & 0 & 0 & 0 & 0 & 0 & 5 & -84 & 1200 & -16854 \\
-4 & 0 & 0 & 0 & 0 & -2 & 28 & -344 & 4360 & -57760 \\
-3 & 0 & 0 & 0 & 1 & -10 & 102 & -1160 & 14274 & -185988 \\
-2 & 0 & 0 & -1 & 4 & -32 & 326 & -3708 & 45722 & -598088 \\
-1 & 0 & 1 & -2 & 12 & -104 & 1085 & -12660 & 159208 & -2112456 \\
1 & 1 & -1 & 5 & -40 & 399 & -4524 & 55771 & -729256 & 9961800 \\
2 & 0 & -1 & 7 & -61 & 648 & -7661 & 97024 & -1293185 & 17921632 \\
3 & 0 & -1 & 9 & -93 & 1070 & -13257 & 173601 & -2371088 & 33470172 \\
4 & 0 & -1 & 12 & -140 & 1750 & -22955 & 312704 & -4396779 & 63460184 \\
5 & 0 & -1 & 15 & -206 & 2821 & -39315 & 559787 & -8136830 & 120497011 \\
\hline
\end{tabular}

Tab.5 Disk degeneracies for brane $I$ or $I I$ in the $O(K) \rightarrow \mathbf{P}^{2}$ geometry.

For the phase $I I I$, to compute the integer invariants we need to change the parameterization of the curve. Consider the $\operatorname{SL}(2, \mathbb{Z})$ transformation

$$
\begin{gathered}
u \rightarrow u^{\prime}=u-v \\
v \rightarrow v^{\prime}=v
\end{gathered}
$$

which will allow us to compute the superpotential in phase III since in these variables, the equation of the phase III leg is $u^{\prime}=0$. The equation for $\Sigma$ written in the new variables becomes

$$
e^{u^{\prime}}+e^{v^{\prime}}+1+e^{-u^{\prime}+3 v^{\prime}-t}=0
$$


upon trivial multiplication by $e^{v^{\prime}}$. The good flat coordinate for this phase is $\hat{v}^{\prime}=$ $v^{\prime}+\Delta_{v}=v^{\prime}+\frac{t-\hat{t}}{3}$. For the integer invariants in phase III we get that $d_{k, m}=0$ for $m<0$ and, for positive $m$, we obtain

\begin{tabular}{|c|rrrrrrrrr|}
\hline$m$ & $k=0$ & 1 & 2 & 3 & 4 & 5 & 6 & 7 & 8 \\
\hline 1 & -1 & 2 & -5 & 32 & -286 & 3038 & -35870 & 454880 & -6073311 \\
2 & 0 & 1 & -4 & 21 & -180 & 1885 & -21952 & 275481 & -3650196 \\
3 & 0 & 1 & -3 & 18 & -153 & 1560 & -17910 & 222588 & -2926959 \\
4 & 0 & 1 & -4 & 20 & -160 & 1595 & -17976 & 220371 & -2869120 \\
5 & 0 & 1 & -5 & 26 & -196 & 1875 & -20644 & 249120 & -3205528 \\
6 & 0 & 1 & -7 & 36 & -260 & 2403 & -25812 & 306095 & -3889116 \\
7 & 0 & 1 & -9 & 52 & -365 & 3254 & -34089 & 397194 & -4981102 \\
8 & 0 & 1 & -12 & 76 & -528 & 4578 & -46812 & 535639 & -6627840 \\
\hline
\end{tabular}

Tab.6 Disk degeneracies for brane III in the $O(K) \rightarrow \mathbf{P}^{2}$ geometry.

From the equation (6.16) we obtain another description of phase $I$, which is at the classical level equivalent to the one already given, but differes in the quantum theory by relative framing $n=-1$, The flat coordinate in the phase $I_{n=-1}$ is

$$
\hat{u}^{\prime}=u^{\prime}
$$

since under the $\mathrm{SL}(2, \mathbb{Z})$ transformation $\Delta_{u}$ and $\Delta_{v}$ cancel off. We have also considered other values of $n$, i.e the D-branes with the $u \rightarrow u^{\prime}=u+n v$ as the dynamical field on the brane. First, note that $I_{n}$ and $I_{-(n+1)}$ (where $I_{n}$ denotes the brane in phase $I$ and with framing $n$ ) are related by $v \rightarrow-v+t$, with $u$ fixed. Thus, we expect

$$
N_{k, m}^{n}= \pm N_{k+m,-m}^{-(n+1)}
$$

where $m$ denotes the is boundary class of the disk. The following are the integer invariants for $n=-1,1,2$, which clearly respect this. 


\begin{tabular}{|c|rrrrrrrrr|}
\hline$n=-1$ & \multicolumn{10}{|c|}{10} & 7 \\
\hline$m$ & $k=0$ & 1 & 2 & 3 & 4 & 5 & 6 & 7 & 8 \\
\hline-5 & 0 & 0 & 0 & 0 & 0 & 40 & -1274 & 27885 & -528934 \\
-4 & 0 & 0 & 0 & 0 & 10 & -253 & 4604 & -76068 & 1214324 \\
-3 & 0 & 0 & 0 & 3 & -54 & 783 & -11058 & 157347 & -2274642 \\
-2 & 0 & 0 & 1 & -13 & 142 & -1657 & 20785 & -274473 & 3769424 \\
-1 & 0 & 1 & -4 & 29 & -274 & 3002 & -36144 & 464522 & -6262370 \\
1 & 1 & -1 & 4 & -29 & 274 & -3002 & 36144 & -464522 & 6262370 \\
2 & 0 & 0 & -1 & 13 & -142 & 1657 & -20785 & 274473 & -3769424 \\
3 & 0 & 0 & 0 & -3 & 54 & -783 & 11058 & -157347 & 2274642 \\
4 & 0 & 0 & 0 & 0 & -10 & 253 & -4604 & 76068 & -1214324 \\
5 & 0 & 0 & 0 & 0 & 0 & -40 & 1274 & -27885 & 528934 \\
\hline
\end{tabular}

\begin{tabular}{|c|c|c|c|c|c|c|c|c|c|c|c|}
\hline$n=1$ & & & & & & $n=2$ & & & & & \\
\hline$m$ & $k=0$ & 1 & 2 & 3 & 4 & $m$ & $k=0$ & 1 & 2 & 3 & 4 \\
\hline-4 & 0 & 0 & 0 & 0 & 0 & -3 & 0 & 0 & 0 & 0 & 0 \\
\hline-3 & 0 & 0 & 0 & 0 & -1 & -2 & 0 & 0 & 0 & -1 & 13 \\
\hline-2 & 0 & 0 & 0 & -1 & 7 & -1 & 1 & -1 & 4 & -29 & 274 \\
\hline-1 & 0 & 1 & -1 & 5 & -40 & 1 & 1 & -4 & 29 & -274 & 3002 \\
\hline 1 & 1 & -2 & 12 & -104 & 1085 & 2 & 1 & -13 & 142 & -1657 & 20785 \\
\hline 2 & -1 & 4 & -32 & 326 & -3708 & 3 & 3 & -54 & 758 & -11058 & 157347 \\
\hline 3 & 1 & -10 & 102 & -1160 & 14274 & 4 & 10 & -253 & 4608 & -76068 & 1214324 \\
\hline 4 & -2 & 28 & -344 & 4360 & -57760 & 5 & 40 & -1274 & 27885 & -528934 & 9380474 \\
\hline
\end{tabular}

Tab.7 Disk degeneracies for brane $I$ in the $O(K) \rightarrow \mathbf{P}^{2}$ geometry for various choice of the ambiguity $n \in Z$.

\section{Integrality of the Bulk Mirror Map}

The integrality of mirror map in the bulk, even though it has been proven in some cases, has not been physically explained. Here we will connect this to the integrality of the number of domain walls $N_{k, m}$. In the case $I_{n=-1}$ we can explicitly show that all coefficients of the form $N_{k, 1}$ are directly related to the coefficients of the bulk mirror map and using this relation the integrality of one follows from the other. 
Define the numbers $a_{i}$ by

$$
e^{\frac{t-\hat{t}}{2}}=1+\sum_{i=1}^{\infty} a_{i} q^{i}
$$

One can show the integrality of $a_{i}$ using the integrality of the mirror map (6.15). Furthermore one can show, by explicitly investigating the Taylor series of $v(\hat{u}, q)$, that

$$
N_{k, 1}=P_{k}\left(a_{1}, \ldots, a_{k-1}\right)-a_{k},
$$

where $P_{k}$ is a polynomial with integer coefficients in the $a_{i}$. E.g. we get

$$
\begin{aligned}
& P_{1}=-3 \\
& P_{2}=30+12 a_{1}+a_{1}^{2} \\
& P_{3}=-420-210 a_{1}-30 a_{1}^{2}-a_{1}^{3}+12 a_{2}+2 a_{1} a_{2} \\
& P_{4}=6930+4200 a_{1}+840 a_{1}^{2}+60 a_{1}^{3}+a_{1}^{4}-210 a_{2}-60 a_{1} a_{2}-3 a_{1}^{2} a_{2}+a_{2}^{2}+12 a_{3}+2 a_{1} a_{3}
\end{aligned}
$$

etc.

The proof that all $P_{k}$ are integer polynomials is tedious and relies on some formulas for the derivatives of $v(\hat{u}, q)$ for this special example. From this one sees that the integrality of $N_{k, 1}$ and $a_{k}$ are equivalent, and the integrality of $a_{k}$ follows from the integrality of the mirror map in the bulk (6.15), and vice-versa. The integrality of mirror map in the bulk had not been physically explained before. Here, by relating it to the integrality of numbers of domain walls $N_{k, 1}$ we have found a physical explanation for it. The integrality of $N_{k, m} m \neq 1$ requires special properties of the $a_{i}$ and seems much more involved.

\section{4. $\mathcal{O}(K) \rightarrow \mathbf{F}_{1}$}

Consider a CY geometry containing the blowup of $\mathbf{P}^{2}$ at one point, which we denote by $\mathbf{F}_{1}$. The charges describing the gauged linear $\sigma$-model on the non-compact Calabi-Yau are $Q_{b}=(-2,0,0,1,1)$ and $Q_{f}=(-1,1,1,-1,0)$, so the corresponding $D$-terms are

$$
\begin{aligned}
\left|X^{3}\right|^{2}+\left|X^{4}\right|^{2}-2\left|X^{0}\right|^{2} & =r_{b} \\
\left|X^{1}\right|^{2}+\left|X^{2}\right|^{2}-\left|X^{0}\right|^{2}-\left|X^{3}\right|^{2} & =r_{f},
\end{aligned}
$$

where $r_{b}$ and $r_{f}$ are the areas of the base and the fiber of the Hirzebruch surface $\mathbf{F}_{1}$. The equations are "solved" in Fig.19. The threefold has two classes of divisors: $H$ coming from the $\mathbb{P}^{2}$ itself and the exceptional divisor $E$ with $H^{2}=1, E^{2}=-1$ and 
$E H=0$. The charge vectors (or generators of the mori-cone) correspond to the fiber $F=H-E$ and the base, which is $E$. Note that $Q_{b}+Q_{f}=Q_{P^{2}}$ with $Q_{P^{2}}$ is the charge for the $O(-3) \rightarrow P^{1}$, and correspondingly $E+F=H$.

The A-brane charges are again such that the brane is special Lagrangian, e.g. $q^{1}=(-1,1,0,0,0)$ and $q^{2}=(-1,0,0,0,1)$ which defines the moduli $c_{1}, c_{2}$ :

$$
\begin{aligned}
& \left|X^{1}\right|^{2}-\left|X^{0}\right|^{2}=c_{1} \\
& \left|X^{4}\right|^{2}-\left|X^{0}\right|^{2}=c_{2}
\end{aligned}
$$

We will consider the following 3 phases (out of a total of 8 possibilities)

$$
\begin{gathered}
\text { Phase I } \quad\left(r_{b}+r_{f}\right) / 2>c_{1}>0 \quad c_{2}=0 \\
\text { Phase II } \quad c_{1}=0 \quad \mathrm{r}_{\mathrm{b}} / 2>\mathrm{c}_{2}>0 \\
\text { Phase III } \quad r_{f} / 2>c_{1}>0 \quad c_{2}=r_{b}
\end{gathered}
$$

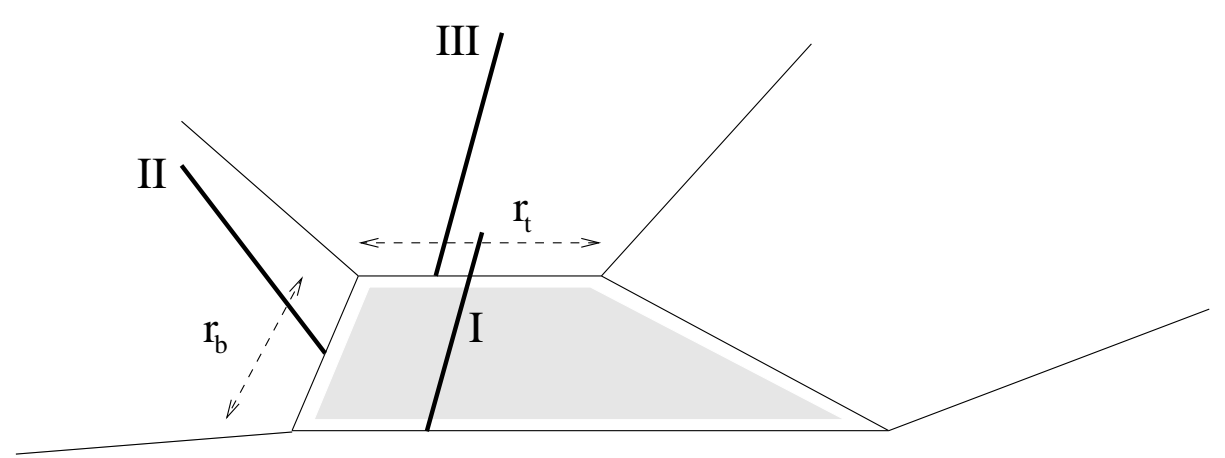

Fig.19 Toric base of $O(K) \rightarrow \mathbf{F}_{\mathbf{1}}$ and the $A$-brane in three phases. $O(K) \rightarrow \mathbf{F}_{\mathbf{1}}$ can be viewed as a blowup of $O(-3) \rightarrow \mathbf{P}^{\mathbf{2}}$ at a point by a $\mathbf{P}^{\mathbf{1}}$ of size $r_{t}$.

The mirror of $\mathcal{O}(K) \rightarrow F_{1}$ is given by

$$
x z=1+e^{u}+e^{-v-u} z_{f} z_{b}+e^{-v} z_{b}+e^{v}
$$

where $z_{b}=e^{-t_{b}}, z_{f}=e^{-t_{f}}$ and we have solved the mirror relations in terms of $Y^{1}=u, Y^{4}=v$. with $Y^{0}=0$. The mirror of the brane in phase $I$ has $u \sim\left(t_{b}+t_{f}\right) / 2$ as a variable with $v \sim 0, I I$ has $v \sim t_{f} / 2$ as a variable with $u \sim 0$ on the relevant leg

8 We have checked that all other phases also lead to integral expansions for disk amplitudes. 
of the toric diagram. One finds using the same methods discussed for the $\mathbf{P}^{1} \times \mathbf{P}^{1}$ example that

$$
\Delta_{u, v}=\left(t_{f}-\hat{t}_{f}\right)+i \pi
$$

The numbers of primitive disks in these two phases, listed side by side are as follows:

\begin{tabular}{|c|rrrrrc|}
\hline$m=-5$ & $I I$ & & & & \\
\hline$k_{b}$ & $k_{f}=0$ & 1 & 2 & 3 & 4 & 5 \\
\hline 5 & 0 & 1 & -6 & 14 & -14 & 5 \\
6 & 0 & 2 & -30 & 140 & -280 & 252 \\
\hline
\end{tabular}

\begin{tabular}{|rccccc|}
\hline$I$ & & & & \\
\hline$k_{f}=0$ & 1 & 2 & 3 & 4 & 5 \\
\hline 0 & 0 & 0 & 0 & 0 & 0 \\
0 & 0 & 0 & 0 & 0 & 42 \\
\hline
\end{tabular}

\begin{tabular}{|c|rrrrrc|}
\hline$m=-4$ & $I I$ & & & & \\
\hline$k_{b}$ & $k_{f}=0$ & 1 & 2 & 3 & 4 & 5 \\
\hline 4 & 0 & 1 & -4 & 5 & -2 & 0 \\
5 & 0 & 2 & -20 & 60 & -70 & 28 \\
6 & 0 & 3 & -68 & 400 & -936 & -344 \\
\hline
\end{tabular}

\begin{tabular}{|rrrrrc|}
\hline$I$ & & & & \\
\hline$k_{f}=0$ & 1 & 2 & 3 & 4 & 5 \\
\hline 0 & 0 & 0 & 0 & -2 & 0 \\
0 & 0 & 0 & 0 & -14 & 28 \\
0 & 0 & 0 & 0 & -52 & 308 \\
\hline
\end{tabular}

\begin{tabular}{|c|rrrrrc|}
\hline$m=-3$ & $I I$ & & & & \\
\hline$k_{b}$ & $k_{f}=0$ & 1 & 2 & 3 & 4 & 5 \\
\hline 3 & 0 & 1 & -2 & 1 & 0 & 0 \\
4 & 0 & 2 & -12 & 20 & -10 & 0 \\
5 & 0 & 3 & -45 & 170 & -230 & 102 \\
6 & 0 & 4 & -130 & 958 & -2612 & 2940 \\
\hline
\end{tabular}

\begin{tabular}{|rrrrrc|}
\hline$I$ & & & & \\
\hline$k_{f}=0$ & 1 & 2 & 3 & 4 & 5 \\
\hline 0 & 0 & 0 & 1 & 0 & 0 \\
0 & 0 & 0 & 5 & -10 & 0 \\
0 & 0 & 0 & 14 & -90 & 102 \\
0 & 0 & 0 & 31 & -450 & 1428 \\
\hline
\end{tabular}

\begin{tabular}{|c|rrrrrc|}
\hline$m=-2$ & $I I$ & & & & \\
\hline$k_{b}$ & $k_{f}=0$ & 1 & 2 & 3 & 4 & 5 \\
\hline 2 & 0 & 1 & -1 & 0 & 0 & 0 \\
3 & 0 & 2 & -6 & 4 & 0 & 0 \\
4 & 0 & 3 & -28 & 57 & -32 & 0 \\
5 & 0 & 4 & -90 & 424 & -664 & 326 \\
6 & 0 & 5 & -237 & 2172 & -6872 & 8640 \\
\hline
\end{tabular}

\begin{tabular}{|rrrrrc|}
\hline$I$ & & & & \\
\hline$k_{f}=0$ & 1 & 2 & 3 & 4 & 5 \\
\hline 0 & 0 & -1 & 0 & 0 & 0 \\
0 & 0 & -2 & 4 & 0 & 0 \\
0 & 0 & -4 & 28 & -32 & 0 \\
0 & 0 & -6 & 112 & -390 & 326 \\
0 & 0 & -9 & 336 & -2500 & 5638 \\
\hline
\end{tabular}




\begin{tabular}{|c|rrrrrc|}
\hline$m=-1$ & $I I$ & & & & \\
\hline$k_{b}$ & $k_{f}=0$ & 1 & 2 & 3 & 4 & 5 \\
\hline 1 & -1 & 1 & 0 & 0 & 0 & 0 \\
2 & 0 & 2 & -2 & 0 & 0 & 0 \\
3 & 0 & 3 & -15 & 12 & 0 & 0 \\
4 & 0 & 4 & -60 & 160 & -104 & 0 \\
5 & 0 & 5 & -175 & 1080 & -1995 & 1085 \\
\hline
\end{tabular}

\begin{tabular}{|rrrrrc|}
\hline$I$ & & & & \\
\hline$k_{f}=0$ & 1 & 2 & 3 & 4 & 5 \\
\hline 0 & 1 & 0 & 0 & 0 & 0 \\
0 & 1 & -2 & 0 & 0 & 0 \\
0 & 1 & -10 & 12 & 0 & 0 \\
0 & 1 & -30 & 120 & -104 & 0 \\
0 & 1 & -70 & 648 & -1596 & 1085 \\
\hline
\end{tabular}

\begin{tabular}{|c|rrrrrc|}
\hline$m=1$ & $I I$ & & & & & \\
\hline$k_{b}$ & $k_{f}=0$ & 1 & 2 & 3 & 4 & 5 \\
\hline 0 & 1 & 0 & 0 & 0 & 0 & 0 \\
1 & 0 & -1 & 0 & 0 & 0 & 0 \\
2 & 0 & -2 & 5 & 0 & 0 & 0 \\
3 & 0 & -3 & 30 & -40 & 0 & 0 \\
4 & 0 & -4 & 105 & -432 & 399 & 0 \\
5 & 0 & -5 & 280 & -2520 & 6370 & -4524 \\
\hline
\end{tabular}

\begin{tabular}{|rrrrcc|}
\hline$I$ & & & & & \\
\hline$k_{f}=0$ & 1 & 2 & 3 & 4 & 5 \\
\hline 1 & 0 & 0 & 0 & 0 & 0 \\
1 & -1 & 0 & 0 & 0 & 0 \\
1 & -6 & 5 & 0 & 0 & 0 \\
1 & -20 & 59 & -40 & 0 & 0 \\
1 & -50 & 356 & -706 & 399 & 0 \\
1 & -105 & 1500 & -6244 & 9372 & -4524 \\
\hline
\end{tabular}

\begin{tabular}{|c|rrrrrc|}
\hline$m=2$ & $I I$ & & & & & \\
\hline$k_{b}$ & $k_{f}=0$ & 1 & 2 & 3 & 4 & 5 \\
\hline 0 & 0 & 0 & 0 & 0 & 0 & 0 \\
1 & 0 & -1 & 0 & 0 & 0 & 0 \\
2 & 0 & -2 & 7 & 0 & 0 & 0 \\
3 & 0 & -3 & 38 & -61 & 0 & 0 \\
4 & 0 & -4 & 128 & -616 & 648 & 0 \\
5 & 0 & -5 & 330 & -3420 & 9744 & -7661 \\
\hline
\end{tabular}

\begin{tabular}{|rrrrrc|}
\hline$I$ & & & & & \\
\hline$k_{f}=0$ & 1 & 2 & 3 & 4 & 5 \\
\hline 0 & 0 & 0 & 0 & 0 & 0 \\
1 & -1 & 0 & 0 & 0 & 0 \\
2 & -9 & 7 & 0 & 0 & 0 \\
4 & -43 & 100 & -61 & 0 & 0 \\
6 & -147 & 756 & -1263 & 648 & 0 \\
9 & -406 & 3920 & -13122 & 17260 & -7661 \\
\hline
\end{tabular}




\begin{tabular}{|c|rrrrrc|}
\hline$m=3$ & $I I$ & & & & & \\
\hline$k_{b}$ & $k_{f}=0$ & 1 & 2 & 3 & 4 & 5 \\
\hline 0 & 0 & 0 & 0 & 0 & 0 & 0 \\
1 & 0 & -1 & 0 & 0 & 0 & 0 \\
2 & 0 & -2 & 9 & 0 & 0 & 0 \\
3 & 0 & -3 & 48 & -93 & 0 & 0 \\
4 & 0 & -4 & 155 & -884 & 1070 & 0 \\
5 & 0 & -5 & 390 & -4682 & 15134 & -13257 \\
\hline
\end{tabular}

\begin{tabular}{|rrrrrc|}
\hline$I$ & 7 & & & & \\
\hline$k_{f}=0$ & 1 & 2 & 3 & 4 & 5 \\
\hline 0 & 0 & 0 & 0 & 0 & 0 \\
1 & -1 & 0 & 0 & 0 & 0 \\
4 & -13 & 9 & 0 & 0 & 0 \\
11 & -85 & 167 & -93 & 0 & 0 \\
25 & -382 & 1555 & -2268 & 1070 & 0 \\
49 & -1344 & 9813 & -27584 & 32323 & -13257 \\
\hline
\end{tabular}

\begin{tabular}{|c|rrrrrc|}
\hline$m=4$ & $I I$ & & & & & \\
\hline$k_{b}$ & $k_{f}=0$ & 1 & 2 & 3 & 4 & 5 \\
\hline 0 & 0 & 0 & 0 & 0 & 0 & 0 \\
1 & 0 & -1 & 0 & 0 & 0 & 0 \\
2 & 0 & -2 & 12 & 0 & 0 & 0 \\
3 & 0 & -3 & 60 & -140 & 0 & 0 \\
4 & 0 & -4 & 188 & -1260 & 1750 & 0 \\
5 & 0 & -5 & 460 & -6397 & 23482 & -22955 \\
\hline
\end{tabular}

\begin{tabular}{|rrrrrc|}
\hline$I$ & & & & & \\
\hline$k_{f}=0$ & 1 & 2 & 3 & 4 & 5 \\
\hline 0 & 0 & 0 & 0 & 0 & 0 \\
1 & -1 & 0 & 0 & 0 & 0 \\
6 & -18 & 12 & 0 & 0 & 0 \\
25 & -155 & 270 & -140 & 0 & 0 \\
76 & -887 & 3056 & -3995 & 1750 & 0 \\
196 & -3873 & 23040 & -56429 & 60021 & -22955 \\
\hline
\end{tabular}

\begin{tabular}{|c|rrrrrr|}
\hline$m=5$ & $I I$ & & & & & \\
\hline$k_{b}$ & $k_{f}=0$ & 1 & 2 & 3 & 4 & 5 \\
\hline 0 & 0 & 0 & 0 & 0 & 0 & 0 \\
1 & 0 & -1 & 0 & 0 & 0 & 0 \\
2 & 0 & -2 & 15 & 0 & 0 & 0 \\
3 & 0 & -3 & 74 & -206 & 0 & 0 \\
4 & 0 & -4 & 225 & -1772 & 2821 & 0 \\
\hline
\end{tabular}

\begin{tabular}{|rrrrrr|}
\hline$I$ & & & & & \\
\hline$k_{f}=0$ & 1 & 2 & 3 & 4 & 5 \\
\hline 0 & 0 & 0 & 0 & 0 & 0 \\
1 & -1 & 0 & 0 & 0 & 0 \\
9 & -24 & 15 & 0 & 0 & 0 \\
49 & -264 & 421 & -206 & 0 & 0 \\
196 & -1876 & 5700 & -6841 & 2821 & 0 \\
\hline
\end{tabular}

Tab.8 Disk degeneracies for brane $I$ and $I I$ in the $O(K) \rightarrow F_{1}$ geometry.

We consider now the phase $I I I$, for which we must change the parameterization of the curve by $u \rightarrow u^{\prime}=-t_{b}-u$, if we are to have the superpotential which is zero classically. In this phase $v$ is the transverse coordinate on the brane. The correction to the flat coordinate is again found by requiring integrality of the amplitude, and we find that

$$
\hat{v}=v+\Delta_{v}=v+t_{f}-\hat{t}_{f}
$$


The disk numbers follow:

\begin{tabular}{|c|rrrrrc|}
\hline$m=1$ & \multicolumn{7}{|c|}{3} & \\
\hline$k_{b}$ & $k_{f}=0$ & 1 & 2 & 3 & 4 & 5 \\
\hline 0 & 1 & -1 & 0 & 0 & 0 & 0 \\
1 & 0 & -2 & 2 & 0 & 0 & 0 \\
2 & 0 & -3 & 15 & -12 & 0 & 0 \\
3 & 0 & -4 & 60 & -160 & 104 & 0 \\
4 & 0 & -5 & 175 & -1080 & 1995 & -1085 \\
5 & 0 & -6 & 420 & -5040 & 19110 & -27144 \\
6 & 0 & -7 & 882 & -18480 & 124033 & -337617 \\
\hline
\end{tabular}

\begin{tabular}{|rrrrrc|}
\hline$m=-1$ & & & & \\
\hline$k_{f}=0$ & 1 & 2 & 3 & 4 & 5 \\
\hline 0 & 0 & 0 & 0 & 0 & 0 \\
-1 & 0 & 0 & 0 & 0 & 0 \\
0 & 1 & 0 & 0 & 0 & 0 \\
0 & 2 & -5 & 0 & 0 & 0 \\
0 & 3 & -30 & 40 & 0 & 0 \\
0 & 4 & -105 & 432 & -399 & 0 \\
0 & 5 & -280 & 2520 & -6370 & 4524 \\
\hline
\end{tabular}

\begin{tabular}{|c|rrrrrc|}
\hline$m=2$ & \multicolumn{1}{|c|}{} \\
\hline$k_{b}$ & $k_{f}=0$ & 1 & 2 & 3 & 4 & 5 \\
\hline 0 & 0 & -1 & 1 & 0 & 0 & 0 \\
1 & 0 & -2 & 6 & -4 & 0 & 0 \\
2 & 0 & -3 & 28 & -57 & 32 & 0 \\
3 & 0 & -4 & 90 & -424 & 664 & -326 \\
4 & 0 & -5 & 237 & -2172 & 6872 & -8640 \\
5 & 0 & -6 & 532 & -8640 & 48208 & -114774 \\
6 & 0 & -7 & 1072 & -28578 & 258516 & -1023679 \\
\hline
\end{tabular}

\begin{tabular}{|rrrrrr|}
\hline$m=-2$ & & & & & \\
\hline$k_{f}=0$ & 1 & 2 & 3 & 4 & 5 \\
\hline 0 & 0 & 0 & 0 & 0 & 0 \\
0 & 0 & 0 & 0 & 0 & 0 \\
0 & 0 & 0 & 0 & 0 & 0 \\
0 & 1 & 0 & 0 & 0 & 0 \\
0 & 2 & -7 & 0 & 0 & 0 \\
0 & 3 & -38 & 61 & 0 & 0 \\
0 & 4 & -128 & 616 & -648 & 0 \\
\hline
\end{tabular}

\begin{tabular}{|c|rrrrrc|}
\hline$m=3$ & \multicolumn{7}{|c|}{} & \multicolumn{1}{c|}{} \\
\hline$k_{b}$ & $k_{f}=0$ & 1 & 2 & 3 & 4 & 5 \\
\hline 0 & 0 & -1 & 2 & -1 & 0 & 0 \\
1 & 0 & -2 & 12 & -20 & 10 & 0 \\
2 & 0 & -3 & 45 & -170 & 230 & -102 \\
3 & 0 & -4 & 130 & -958 & 2612 & -2940 \\
4 & 0 & -5 & 315 & -4116 & 19750 & -41996 \\
5 & 0 & -6 & 672 & -14520 & 112970 & -398970 \\
6 & 0 & -7 & 1302 & -44073 & 525031 & -2854610 \\
\hline
\end{tabular}

\begin{tabular}{|rrrrrr|}
\hline$m=-3$ & & & & & \\
\hline$k_{f}=0$ & 1 & 2 & 3 & 4 & 5 \\
\hline 0 & 0 & 0 & 0 & 0 & 0 \\
0 & 0 & 0 & 0 & 0 & 0 \\
0 & 0 & 0 & 0 & 0 & 0 \\
0 & 0 & 0 & 0 & 0 & 0 \\
0 & 1 & 0 & 0 & 0 & 0 \\
0 & 2 & -9 & 0 & 0 & 0 \\
0 & 3 & -48 & 93 & 0 & 0 \\
\hline
\end{tabular}




\begin{tabular}{|c|rrrrrr|}
\hline$m=4$ & \multicolumn{10}{|c|}{} \\
\hline$k_{b}$ & $k_{f}=0$ & 1 & 2 & 3 & 4 & 5 \\
\hline 0 & 0 & -1 & 4 & -5 & 2 & 0 \\
1 & 0 & -2 & 20 & -60 & 70 & -28 \\
2 & 0 & -3 & 68 & -400 & 936 & -945 \\
3 & 0 & -4 & 180 & -1912 & 7910 & -15030 \\
4 & 0 & -5 & 412 & -7290 & 49096 & -155035 \\
5 & 0 & -6 & 840 & -23520 & 243558 & -1185830 \\
6 & 0 & -7 & 1576 & -66660 & 1015960 & -7246659
\end{tabular} \mid \begin{tabular}{rrrrrrr|}
$m=-4$ \\
$k_{f}=0$ & 1 & 2 & 3 & 4 & 5 \\
\hline 0 & 0 & 0 & 0 & 0 & 0 \\
0 & 0 & 0 & 0 & 0 & 0 \\
0 & 0 & 0 & 0 & 0 & 0 \\
0 & 0 & 0 & 0 & 0 & 0 \\
0 & 0 & 0 & 0 & 0 & 0 \\
0 & 1 & 0 & 0 & 0 & 0 \\
0 & 2 & -12 & 0 & 0 & 0 \\
\hline
\end{tabular}

\begin{tabular}{|c|c|c|c|c|c|c|c|c|c|c|c|c|}
\hline$m=5$ & & & & & & & $m=-5$ & & & & & \\
\hline$k_{b}$ & $k_{f}=0$ & 1 & 2 & 3 & 4 & 5 & $k_{f}=0$ & 1 & 2 & & 3 & 45 \\
\hline 0 & 0 & -1 & 6 & -14 & 14 & -5 & 0 & 0 & & ) & 0 & $\begin{array}{ll}0 & 0\end{array}$ \\
\hline 1 & 0 & -2 & 30 & -140 & 280 & -252 & 0 & 0 & & ) & 0 & $\begin{array}{ll}0 & 0\end{array}$ \\
\hline 2 & 0 & -3 & 95 & -810 & 2870 & -4858 & 0 & 0 & & ) & 0 & $\begin{array}{ll}0 & 0\end{array}$ \\
\hline 3 & 0 & -4 & 240 & -3472 & 20150 & -56728 & 0 & 0 & & ) & 0 & $\begin{array}{ll}0 & 0\end{array}$ \\
\hline 4 & 0 & -5 & 525 & -12156 & 109167 & -475047 & 0 & 0 & & ) & 0 & $\begin{array}{ll}0 & 0\end{array}$ \\
\hline 5 & 0 & -6 & 1036 & -36648 & 487382 & -3116370 & 0 & 0 & & ) & 0 & $0 \quad 0$ \\
\hline 6 & 0 & -7 & 1890 & -98340 & 1869595 & -16909871 & 0 & 1 & & & 0 & $\begin{array}{ll}0 & 0\end{array}$ \\
\hline
\end{tabular}

Tab.9 The disk degeneracies for brane $I I I$ in the $O(K) \rightarrow F_{1}$.

\section{Acknowledgements}

We are grateful to Volker Braun, Stefan Fredenhagen, Kentaro Hori, Amer Iqbal, Dominic Joyce, Sheldon Katz, Chiu-Chu Liu, Marcos Marino, Cliff Taubes, ShingTung Yau and Eric Zaslow for very valuable discussions.

The research of M.A. and C.V. is supported in part by NSF grants PHY-9802709 and DMS 9709694. The research of A.K. is supported in part by the GIF grant I645-130.14/1999.

\section{Appendix A. Large $N$ Limit of Chern-Simons and Framing of the Knot}

As discussed in section 5, Wilson loop observables of Chern-Simons theory at large $N$ are naturally encoded in terms of the expectation values

$$
\left\langle\exp \sum_{n} \frac{1}{n} \operatorname{tr}^{\mathrm{n}} \operatorname{tr}^{\mathrm{n}}\right\rangle=\exp \left(-\mathrm{F}\left(\mathrm{V}, \mathrm{t}, \mathrm{g}_{\mathrm{s}}\right)\right)
$$


where $V$ is viewed as a source and $U$ is the holonomy of the connection along the loop. If we are interested in extracting the amplitudes with a single hole, we consider terms on the right hand side of the form $\operatorname{trV}^{\mathrm{n}}$ in the exponent. This will correspond to contributions where the large $N$ worldsheet wraps $n$ times around the loop. From the left-hand side this is equivalent to computation of

$$
\left\langle\frac{1}{n} \operatorname{tr}^{\mathrm{n}}\right\rangle
$$

For a special choice of framing of the unknot this was computed in [4 and it was shown that

$$
\left\langle\frac{1}{n} \operatorname{tr} \mathrm{U}^{\mathrm{n}}\right\rangle=\frac{1}{\mathrm{n}} \operatorname{tr} \mathrm{U}_{0}^{\mathrm{n}}
$$

where $U_{0}$ is a particular element of $S U(N)$ given by a diagonal matrix with entries $\exp \left(\frac{i \pi(N+1-2 r)}{N+k}\right)$, as $r$ ranges from 1 to $N$. This leads, in the leading order in $g_{s}$, corresponding to disk amplitude ( $g=0$ and one hole), to

$$
\left\langle\frac{1}{n} \operatorname{trU}^{\mathrm{n}}\right\rangle=\frac{1}{\mathrm{n}^{2} \mathrm{~g}_{\mathrm{s}}}[\exp (\mathrm{nt} / 2)-\exp (-\mathrm{nt} / 2)]
$$

where in the large $t$ limit and by redefining the $V$ (and absorbing a factor of $\exp (t / 2$ ) in it ) and in the large $t$ limit, we obtain the $\mathbf{C}^{3}$ answer for the disk amplitude

$$
\frac{1}{g_{s}} \sum_{n} \frac{1}{n^{2}} \operatorname{trV}^{\mathrm{n}}
$$

(where we have identified in this paper $V=e^{u}$ ).

Now we ask what happens if we choose a different framing. For the expectation value of the Wilson loops with holonomies in a given representation $R$ of $S U(N)$ the answer is relatively simple:

$$
\left\langle\operatorname{Tr}_{\mathrm{R}} \mathrm{U}\right\rangle_{\mathrm{p}}=\left\langle\operatorname{Tr}_{\mathrm{R}} \mathrm{U}\right\rangle_{0} \exp \left(2 \pi \mathrm{ip} \frac{\mathrm{h}_{\mathrm{R}}}{\mathrm{k}+\mathrm{N}}\right)
$$

where $p$ denotes the change in the framing from a given one denoted by 0 (characterized by an element of topological class of winding of an $S^{1}$ over an $S^{1}$ ), and $h_{R}$ denotes the Casimir of the representation. In other words, the result of the change in framing is a multiplication by $\exp \left(g_{s} p h_{R}\right)$.

However we need to compute the correlation function for $\left\langle\operatorname{tr} \mathrm{U}^{\mathrm{n}}\right\rangle$ which is the trace in the fundamental representation of $U^{n}$. To find the change in this correlation function due to change in framing, we will first have to write it in terms of trace 
of $U$ in different representations, compute each one, and multiply each one with $\exp \left(g_{s} p h_{R}\right)$.

There is an identity which is useful for this purpose:

$$
\operatorname{tr} \mathrm{U}^{\mathrm{n}}=\sum_{\mathrm{s}=0}^{\mathrm{n}-1}(-1)^{\mathrm{s}} \operatorname{Tr}_{\mathrm{R}_{\mathrm{n}, \mathrm{s}}} \mathrm{U}
$$

where $R_{n, s}$ denotes representations of $S U(N)$ with $n$ boxes for the Young Tableau which look like ' $\Gamma$ ' and which consists of only one non-trivial row and one non-trivial column. $s+1$ denotes the number of elements in the first column.

Combining all this we find that the coefficient of $\operatorname{tr} V^{n}$ for the unknot and with framing number $p$ is given by

$$
\left\langle\frac{1}{n} \operatorname{tr} \mathrm{U}^{\mathrm{n}}\right\rangle=\frac{1}{\mathrm{n}} \sum_{\mathrm{s}=0}^{\mathrm{n}-1}(-1)^{\mathrm{s}} \exp \left(\operatorname{pg}_{\mathrm{s}} \mathrm{h}_{\mathrm{R}_{\mathrm{n}, \mathrm{s}}}\right) \operatorname{Tr}_{\mathrm{R}_{\mathrm{n}, \mathrm{s}}} \mathrm{U}_{0}
$$

We are interested in extracting the disk amplitude which is the leading term for this expansion as $g_{s} \rightarrow 0$. Moreover we will be interested in the limit where $t \rightarrow \infty$, but where we rescale $V$ by a factor of $e^{t / 2}$ so that we would be computing the brane in the $\mathbf{C}^{3}$ geometry. The most complicated aspect of this computation is finding $\operatorname{Tr}_{\mathrm{R}_{\mathrm{n}, \mathrm{s}}} \mathrm{U}_{0}$. We will reduce this computation to a group theory computation as follows: Let

$$
\operatorname{Tr}_{\mathrm{R}_{\mathrm{n}, \mathrm{s}}} \mathrm{U}_{0}=\sum_{\sum \mathrm{in}_{\mathrm{i}}=\mathrm{n}} \alpha_{\mathrm{n}_{\mathrm{i}}}^{\mathrm{R}_{\mathrm{n}, \mathrm{s}}} \prod_{\mathrm{i}=1}^{\mathrm{n}}\left[\operatorname{trU}_{0}^{\mathrm{i}}\right]^{\mathrm{n}_{\mathrm{i}}}
$$

for some group theoretic factor $\alpha_{n_{i}}^{R_{n, s}}$. Using this and the leading computation at large $N$ for the unknot with the standard framing we can reduce the above computation to

$$
\left\langle\frac{1}{n} \operatorname{tr} U^{\mathrm{n}}\right\rangle=\frac{1}{\mathrm{n}} \sum_{\mathrm{s}=0}^{\mathrm{n}-1}(-1)^{\mathrm{s}} \exp \left(\operatorname{pg}_{\mathrm{s}} \mathrm{h}_{\mathrm{R}_{\mathrm{n}, \mathrm{s}}}\right) \sum_{\sum \mathrm{in}_{\mathrm{i}}=\mathrm{n}} \alpha_{\mathrm{n}_{\mathrm{i}}}^{\mathrm{R}_{\mathrm{n}, \mathrm{s}}} \prod_{\mathrm{i}=1}^{\mathrm{n}}\left(\frac{1}{\mathrm{ig}_{\mathrm{s}}}\right)^{\mathrm{n}_{\mathrm{i}}}
$$

Using this and the fact that up to a further redefinition of $V$ and dropping a subleading term in $g_{s}, h_{R_{n, s}}=\frac{1}{2} n(n-1-2 s)$ we have computed the above expression for $n=1,2,3$ and found perfect match with the result of the computation done for $\mathbf{C}^{3}$ when we shift $u \rightarrow u+p v$. Moreover we have verified that we obtain for all $n$ a polynomial of degree $n-1$ in $p$ in agreement with the results of $\mathbf{C}^{3}$. Furthermore we have verified $\mathrm{\theta}$ that the leading power in $p$ agrees between both computations for all $n$.

9 This involves using the identity $\frac{n^{n-2}}{n ! 2^{n-1}} \sum_{r=1}^{n}(-1)^{r+1} \frac{(n-2 r+1)^{n-1}}{(n-r) !(r-1) !}=\frac{n^{n-2}}{n !}$. 


\section{References}

[1] M. Bershadsky, S. Cecotti, H. Ooguri and C. Vafa, "Kodaira-Spencer theory of gravity and exact results for quantum string amplitudes," Commun. Math. Phys. 165, 311 (1994), hep-th/9309140.

[2] I. Brunner, M.R. Douglas, A. Lawrence and C. Römelsberger, "D-branes on the quintic," JHEP 0008, 015 (2000), hep-th/9906200.

[3] S. Kachru, S. Katz, A. E. Lawrence and J. McGreevy, "Open string instantons and superpotentials, Phys. Rev. D 62, 026001 (2000), hep-th/9912151; "Mirror symmetry for open strings," Phys. Rev. D 62 (2000) 126005, hep-th/0006047.

[4] H. Ooguri and C. Vafa, "Knot invariants and topological strings," Nucl. Phys. B 577, 419 (2000), hep-th/9912123.

[5] C. Vafa, "Superstrings and Topological Strings at Large $N$," hep-th/0008142.

[6] M. Aganagic and C. Vafa, "Mirror symmetry, D-branes and counting holomorphic discs," hep-th/0012041.

[7] R. Gopakumar and C. Vafa, "On the gauge theory/geometry correspondence," Adv. Theor. Math. Phys. 3, 1415 (1999), hep-th/9811131.

[8] E. Witten, "Quantum Field Theory and the Jones Polynomial," Commun. Math. Phys. 121, (1989) 351-399.

[9] E. Witten, "Phases of $\mathrm{N}=2$ theories in two dimensions," Nucl. Phys. B 403, 159 (1993), hep-th/9301042.

[10] K. Hori and C. Vafa, "Mirror symmetry," hep-th/0002222.

[11] K. Hori, "Linear Models of Supersymmetric D-Branes," hep-th/0012179.

[12] D. Joyce, "On counting special Lagrangian homology 3-spheres," hep-th/9907013.

[13] T. R. Taylor and C. Vafa, "RR flux on Calabi-Yau and partial supersymmetry breaking," Phys. Lett. B 474, 130 (2000), hep-th/9912152.

[14] P. Mayr, "On supersymmetry breaking in string theory and its realization in brane worlds," Nucl. Phys. B 593, 99 (2001), hep-th/0003198.

[15] G. Curio, A. Klemm, D. Lüst and S. Theisen, "On the vacuum structure of type II string compactifications on Calabi-Yau spaces with H-fluxes," hep-th/0012213.

[16] B. S. Acharya, "On Realising $N=1$ Super Yang-Mills in M theory," hepth/0011089.

[17] M. Atiyah, J. Maldacena and C. Vafa, "An M-theory flop as a large $N$ duality," hep-th/0011256.

[18] J. Gomis, "D-branes, holonomy and M-theory," hep-th/0103115.

[19] J. D. Edelstein and C. Nunez, "D6 branes and M-theory geometrical transitions from gauged supergravity," hep-th/0103167.

[20] S. Kachru and J. McGreevy, "M-theory on manifolds of G(2) holonomy and type IIA orientifolds," hep-th/0103223. 
[21] J. A. Harvey and G. Moore, "Superpotentials and membrane instantons," hepth/9907026.

[22] C. Vafa, "Extending Mirror Conjecture to Calabi-Yau with Bundles," hepth/9804131.

[23] J. H. Schwarz, "The power of M theory," Phys. Lett. B 367, 97 (1996), hepth/9510086.

[24] N. C. Leung and C. Vafa, "Branes and toric geometry," Adv. Theor. Math. Phys. 2, 91 (1998), hep-th/9711013.

[25] O. Aharony, A. Hanany and B. Kol, "Webs of (p,q) 5-branes, five dimensional field theories and grid diagrams," JHEP 9801, 002 (1998) hep-th/9710116.

[26] J. M. Labastida, M. Marino and C. Vafa, "Knots, links and branes at large N," JHEP 0011, 007 (2000), hep-th/0010102.

[27] J. M. Labastida and M. Marino, "Polynomial invariants for torus knots and topological strings," Commun. Math. Phys. 217, 423 (2001), hep-th/0004196.

[28] R. Gopakumar and C. Vafa, "M-theory and topological strings I and II," hepth/9809187 and hep-th/9812127.

[29] E. Witten, "Chern-Simons gauge theory as a string theory," hep-th/9207094.

[30] C. Taubes, To appear.

[31] I. S. Gradshteyn and I. M. Ryzhik, "Table of Integrals, Series and Products," Academic Press, Boston (1994).

[32] S. Katz and C.-C. M. Liu, "Enumerative Geometry of Stable Maps with Lagrangian Boundary Conditions and Multiple Covers of the Disc," math.AG/0103074.

[33] J. Li and Y. S. Song, "Open String Instantons and Relative Stable Morphisms," hep-th/0103100.

[34] T. M. Chiang, A. Klemm, S. T. Yau and E. Zaslow, "Local mirror symmetry: Calculations and interpretations," Adv. Theor. Math. Phys. 3 (1999) 495, hepth/9903053.

[35] M. Aganagic, A. Karch, D. Lust and A. Miemiec, "Mirror Symmetries for Brane Configurations and Branes at Singularities," hep-th/9903093.

[36] K. Hori, A. Iqbal and C. Vafa, "D-Branes And Mirror Symmetry," hepth/0005247.

[37] A. Klemm and E. Zaslow, "Local mirror symmetry at higher genus," hepth/9906046. 